\title{
A NEW APPROACH TO KY FAN-TYPE INEQUALITIES
}

PENG GAO

Received 12 May 2005 and in revised form 20 October 2005

The study of the behavior of means under equal increments of their variables provides a new approach to Ky Fan-type inequalities. Via this approach we are able to prove some new results on Ky Fan-type inequalities. We also prove some inequalities involving the symmetric means.

\section{Introduction}

Let $M_{n, r}(\mathbf{x})$ be the generalized weighted power means: $M_{n, r}(\mathbf{x})=\left(\sum_{i=1}^{n} \omega_{i} x_{i}^{r}\right)^{1 / r}$, where $\omega_{i}>0,1 \leq i \leq n$, with $\sum_{i=1}^{n} \omega_{i}=1$ and $\mathbf{x}=\left(x_{1}, x_{2}, \ldots, x_{n}\right)$. Here $M_{n, 0}(\mathbf{x})$ denotes the limit of $M_{n, r}(\mathbf{x})$ as $r \rightarrow 0^{+}$. Unless specified otherwise, we always assume $0<x_{1} \leq x_{2} \cdots \leq x_{n}$. We denote $\sigma_{n}=\sum_{i=1}^{n} \omega_{i}\left(x_{i}-A_{n}\right)^{2}$.

To any given $\mathbf{x}, t \geq 0$ we associate $\mathbf{x}^{\prime}=\left(1-x_{1}, 1-x_{2}, \ldots, 1-x_{n}\right), \mathbf{x}_{t}=\left(x_{1}+t, \ldots, x_{n}+t\right)$. When there is no risk of confusion, we will write $M_{n, r}$ for $M_{n, r}(\mathbf{x}), M_{n, r, t}$ for $M_{n, r}\left(\mathbf{x}_{t}\right)$, and $M_{n, r}^{\prime}$ for $M_{n, r}\left(\mathbf{x}^{\prime}\right)$ if $x_{n}<1$. We also define $A_{n}=M_{n, 1}, G_{n}=M_{n, 0}, H_{n}=M_{n,-1}$ and similarly for $A_{n}^{\prime}, G_{n}^{\prime}, H_{n}^{\prime}, A_{n, t}, G_{n, t}, H_{n, t}$.

To simplify expressions, we define

$$
\Delta_{r, s, t, \alpha}=\frac{M_{n, r, t}^{\alpha}-M_{n, s, t}^{\alpha}}{M_{n, r}^{\alpha}-M_{n, s}^{\alpha}}, \quad \Delta_{r, s}^{\prime}=\frac{M_{n, r}^{\prime}-M_{n, s}^{\prime}}{M_{n, r}-M_{n, s}}
$$

with $\Delta_{r, s, t, 0}=\left(\ln \left(M_{n, r, t} / M_{n, s, t}\right)\right) /\left(\ln \left(M_{n, r} / M_{n, s}\right)\right)$. We also write $\Delta_{r, s, t}$ for $\Delta_{r, s, t, 1}$. In order to include the case of equality for various inequalities in our discussions, for any given inequality, we define $0 / 0$ to be the number which makes the inequality an equality.

Recently, the author [14, Theorem 2.1] has proved the following result.

Theorem 1.1. For $r>s$, the following inequalities are equivalent:

$$
\begin{gathered}
\frac{r-s}{2 x_{1}} \sigma_{n} \geq M_{n, r}-M_{n, s} \geq \frac{r-s}{2 x_{n}} \sigma_{n}, \\
\frac{x_{n}}{1-x_{n}} \geq \Delta_{r, s}^{\prime} \geq \frac{x_{1}}{1-x_{1}},
\end{gathered}
$$

where in (1.3) we require $x_{n}<1$. 
Cartwright and Field [9] first proved the validity of (1.2) for $r=1, s=0$. For other extensions and refinements of $(1.2)$, see $[3,13,18,19]$. Inequality (1.3) is commonly referred to as the additive Ky Fan's inequality. We refer the reader to the survey article [2] and the references therein for an account of Ky Fan's inequality.

The study of the behavior of means under equal increments of their variables was initiated by Hoehn and Niven [16]. Aczél and Páles [1] proved $\Delta_{1, s, t} \leq 1$ for any $s \neq 1$. We can interpret their result as an assertion of the monotonicity of $A_{n, t}-M_{n, s, t}$ as a function of $t$. The asymptotic behavior of $t\left(M_{n, r, t}-A_{n, t}\right)$ was studied by Brenner and Carlson [7]. The same idea of [14] can be used to show that both (1.2) and (1.3) are equivalent to

$$
\frac{x_{n}}{t+x_{1}} \geq \Delta_{r, s, t} \geq \frac{x_{1}}{t+x_{n}}
$$

which holds for all $t \geq 0$,

In Section 3, we will study the monotonicities of $\left(t+x_{n}\right)\left(M_{n, r, t}-M_{n, s, t}\right)$ and $(t+$ $\left.x_{1}\right)\left(M_{n, r, t}-M_{n, s, t}\right)$ as functions of $t$ for $r=1$ or $s=1$ and then apply the result to inequalities of the type (1.2).

The study of the behavior of means under equal increments of their variables provides us with a new approach of studying Ky Fan-type inequalities. In Section 4, we use this approach to show that some of the inequalities we have studied are actually equivalent.

The following inequality connecting three classical means (with $\omega_{i}=1 / n$ here) is due to W. L. Wang and P. F. Wang [24] (left-hand side inequality) and Alzer et al. [5] (righthand side inequality):

$$
\left(\frac{A_{n}}{A_{n}^{\prime}}\right)^{n-1} \frac{H_{n}}{H_{n}^{\prime}} \geq\left(\frac{G_{n}}{G_{n}^{\prime}}\right)^{n} \geq\left(\frac{H_{n}}{H_{n}^{\prime}}\right)^{n-1} \frac{A_{n}}{A_{n}^{\prime}}
$$

The above inequality was refined in [14] and in Section 5 we will give another refinement of the above inequality.

Alzer [4] has given a counterexample to show that $A_{n}^{\alpha}-G_{n}^{\alpha}$ and $A_{n}^{\prime \alpha}-G_{n}^{\prime \alpha}$ are not comparable in general for $\alpha>1$. However, Pečarić and Alzer [22] (see also [2, Theorem 7.2] proved the following result.

Theorem 1.2. For $\omega_{i}=1 / n, x_{n} \leq 1 / 2$,

$$
A_{n}^{\prime n}-G_{n}^{\prime n} \geq A_{n}^{n}-G_{n}^{n}
$$

Theorem 1.2 suggests that $A_{n}^{\prime \alpha}-G_{n}^{\prime \alpha} \geq A_{n}^{\alpha}-G_{n}^{\alpha}$ for $\alpha=1 / q$ with $q=\min \left\{\omega_{i}\right\}$, a result we will establish in Section 6. A similar result is also proved there.

Let $r \in\{0,1, \ldots, n\}$; the $r$ th symmetric function $E_{n, r}$ of $\mathbf{x}$ and its mean $P_{n, r}$ are defined by

$$
E_{n, r}(\mathbf{x})=\sum_{1 \leq i_{1}<\cdots<i_{r} \leq n} \prod_{j=1}^{r} x_{i_{j}}, \quad 1 \leq r \leq n, \quad E_{n, 0}=1, \quad P_{n, r}^{r}(\mathbf{x})=\frac{E_{n, r}(\mathbf{x})}{\left(\begin{array}{l}
n \\
r
\end{array}\right)} .
$$

The usage of $E_{n, r}, E_{n, r}^{\prime}, E_{n, r, t}, P_{n, r}, P_{n, r}^{\prime}, P_{n, r, t}$ is similar to the case for the power means. 
Many of the results we will obtain for power means also have their analogues for symmetric means, which we will spend the last section to explore. For example, the result of W. L. Wang and P. F. Wang mentioned above is more general; they have shown the following.

Theorem 1.3. For $1 \leq r \leq n-1, x_{i} \in(0,1 / 2], 1 \leq i \leq n$,

$$
\ln P_{n, r}-\ln P_{n, r+1} \geq \ln P_{n, r}^{\prime}-\ln P_{n, r+1}^{\prime} .
$$

We also note the following result of Bullen and Marcus [8].

Theorem 1.4. For $1 \leq k \leq r \leq n$,

$$
(r+1)\left(\ln P_{n+1, k}-\ln P_{n+1, r+1}\right) \geq r\left(\ln P_{n, k}-\ln P_{n, r}\right)
$$

with equality holding if and only if $x_{1}=\cdots=x_{n+1}$.

In Section 7, we will provide a refinement of Theorems 1.3 and 1.4 for $k=1$.

\section{Lemmas}

Lemma 2.1. Let $J(x)$ be the smallest closed interval that contains all of $x_{i}$ and let $f(x), g(x) \in$ $C^{2}(J(x))$ be two twice differentiable functions, then

$$
\frac{\sum_{i=1}^{n} \omega_{i} f\left(x_{i}\right)-f\left(\sum_{i=1}^{n} \omega_{i} x_{i}\right)}{\sum_{i=1}^{n} \omega_{i} g\left(x_{i}\right)-g\left(\sum_{i=1}^{n} \omega_{i} x_{i}\right)}=\frac{f^{\prime \prime}(\xi)}{g^{\prime \prime}(\xi)}
$$

for some $\xi \in J(x)$, provided that the denominator of the left-hand side is nonzero.

Lemma 2.1 and the following consequence of it are due to Mercer [17].

Lemma 2.2. For $w>u, w \neq v, u \neq v$,

$$
\left|\frac{u(u-v)}{w(w-v)}\right| \frac{1}{x_{1}^{w-u}} \geq\left|\frac{\left(M_{n, u}^{u}-M_{n, v}^{u}\right)}{\left(M_{n, w}^{w}-M_{n, v}^{w}\right)}\right| \geq\left|\frac{u(u-v)}{w(w-v)}\right| \frac{1}{x_{n}^{w-u}}
$$

with equality holding if and only if $x_{1}=\cdots=x_{n}$.

Lemma 2.3. Let $t \geq 0, \mathbf{q}=\left(q_{1}, \ldots, q_{n}\right)$ with $q_{i} \geq 1,1 \leq i \leq n$, then for $n \geq 2, f(\mathbf{x} ; \mathbf{q}) \leq$ $f\left(\mathbf{x}_{t} ; \mathbf{q}\right)$, where

$$
f(\mathbf{x} ; \mathbf{q})=x_{n}^{2}\left(\frac{\left(\sum_{i=1}^{n} q_{i}\right)-1}{\sum_{i=1}^{n} q_{i} x_{i}}+\frac{1}{x_{1}^{2}\left(\sum_{i=1}^{n} q_{i} / x_{i}\right)}-\frac{1}{x_{1}}\right),
$$


3554 A new approach to Ky Fan-type inequalities

and equality holds if and only if one of the following conditions holds: (i) $t=0$; (ii) $n=2$, $q_{1}=q_{2}=1$; (iii) $x_{1}=x_{2}=\cdots=x_{n}$. Moreover, when (ii) or (iii) happens, it also holds that $f(\mathbf{x} ; \mathbf{q})=f\left(\mathbf{x}_{t} ; \mathbf{q}\right)=0$.

Proof. It is routine to check $f(\mathbf{x} ; \mathbf{q})=f\left(\mathbf{x}_{t} ; \mathbf{q}\right)$ when $t=0$ holds. So now we may assume that $t>0$. Then one checks easily that

$$
\begin{aligned}
& \frac{\left(\sum_{i=1}^{n} q_{i}\right)-1}{\sum_{i=1}^{n} q_{i} x_{i}}-\frac{1}{x_{1}}+\frac{1}{x_{1}^{2}\left(\sum_{i=1}^{n} q_{i} / x_{i}\right)} \\
& \quad=\frac{\sum_{i=1}^{n} q_{i}\left(x_{1}-x_{i}\right)-x_{1}}{x_{1}\left(\sum_{i=1}^{n} q_{i} x_{i}\right)}+\frac{1}{x_{1}^{2}\left(\sum_{i=1}^{n} q_{i} / x_{i}\right)} \\
& =\frac{\left(\sum_{i=1}^{n} q_{i}\left(x_{1}-x_{i}\right)-x_{1}\right) x_{1}\left(\sum_{i=1}^{n} q_{i} / x_{i}\right)+\sum_{i=1}^{n} q_{i} x_{i}}{x_{1}^{2}\left(\sum_{i=1}^{n} q_{i} x_{i}\right)\left(\sum_{i=1}^{n} q_{i} / x_{i}\right)} .
\end{aligned}
$$

Note that

$$
\begin{aligned}
\left(\sum_{i=1}^{n} q_{i}\left(x_{1}-x_{i}\right)-x_{1}\right) x_{1}\left(\sum_{i=1}^{n} \frac{q_{i}}{x_{i}}\right)+\sum_{i=1}^{n} q_{i} x_{i} \\
=\left(\sum_{i=1}^{n} q_{i}\left(x_{1}-x_{i}\right)\right) x_{1}\left(\sum_{i=1}^{n} \frac{q_{i}}{x_{i}}\right)-\sum_{i=1}^{n} \frac{q_{i}\left(x_{1}^{2}-x_{i}^{2}\right)}{x_{i}} \\
=\sum_{i=1}^{n} q_{i}\left(x_{1}-x_{i}\right)\left(\left(q_{1}-1\right)+\sum_{j \neq 1, i} \frac{q_{j} x_{1}}{x_{j}}+\frac{\left(q_{i}-1\right) x_{1}}{x_{i}}\right) .
\end{aligned}
$$

Hence,

$$
f(\mathbf{x} ; \mathbf{q})=\frac{\sum_{i=1}^{n} q_{i}\left(x_{1}-x_{i}\right)\left(\left(q_{1}-1\right) x_{n} / x_{1}+\sum_{j \neq 1, i}\left(q_{j} x_{n} / x_{j}\right)+\left(q_{i}-1\right) x_{n} / x_{i}\right)}{\left(\sum_{i=1}^{n} q_{i} x_{i} / x_{n}\right)\left(\sum_{i=1}^{n} q_{i} x_{1} / x_{i}\right)} \leq 0 .
$$

Since $x / y>(x+t) /(y+t)$ when $x>y>0, t>0$, it follows from the above expression that $f(\mathbf{x} ; \mathbf{q}) \leq f\left(\mathbf{x}_{t} ; \mathbf{q}\right)$. The conditions for equality can be checked easily and this completes the proof.

Lemma 2.4. Let $t \geq 0, \mathbf{q}=\left(q_{1}, \ldots, q_{n}\right)$ with $q_{i} \geq 1,1 \leq i \leq n$, then for $n \geq 2, g(\mathbf{x} ; \mathbf{q}) \leq$ $g\left(\mathbf{x}_{t} ; \mathbf{q}\right)$, where

$$
g(\mathbf{x} ; \mathbf{q})=x_{n}^{2}\left(\frac{1}{x_{1}}-\frac{1}{\sum_{i=1}^{n} q_{i} x_{i}}-\frac{\left(\sum_{i=1}^{n} q_{i}\right)-1}{x_{1}^{2}\left(\sum_{i=1}^{n} q_{i} / x_{i}\right)}\right)
$$

and equality holds if and only if one of the following conditions holds: (i) $t=0$; (ii) $n=2$, $q_{1}=q_{2}=1$; (iii) $x_{1}=x_{2}=\cdots=x_{n}$. Moreover, when (ii) or (iii) happens, it also holds that $g(\mathbf{x} ; \mathbf{q})=g\left(\mathbf{x}_{t} ; \mathbf{q}\right)=0$. 
Proof. It is routine to check $g(\mathbf{x} ; \mathbf{q})=g\left(\mathbf{x}_{t} ; \mathbf{q}\right)$ when $t=0$ holds. So now we may assume that $t>0$. Then one checks easily that

$$
\begin{aligned}
\frac{1}{x_{1}}- & \frac{\left(\sum_{i=1}^{n} q_{i}\right)-1}{x_{1}^{2}\left(\sum_{i=1}^{n} q_{i} / x_{i}\right)}-\frac{1}{\sum_{i=1}^{n} q_{i} x_{i}} \\
= & \frac{\sum_{i=1}^{n} q_{i}\left(x_{1}-x_{i}\right) / x_{i}+1}{x_{1}^{2}\left(\sum_{i=1}^{n} q_{i} / x_{i}\right)}-\frac{1}{\sum_{i=1}^{n} q_{i} x_{i}} \\
= & \frac{\left(\sum_{i=1}^{n} q_{i}\left(x_{1}-x_{i}\right) / x_{i}+1\right)\left(\sum_{i=1}^{n} q_{i} x_{i}\right)-x_{1}^{2}\left(\sum_{i=1}^{n} q_{i} / x_{i}\right)}{x_{1}^{2}\left(\sum_{i=1}^{n} q_{i} x_{i}\right)\left(\sum_{i=1}^{n} q_{i} / x_{i}\right)} .
\end{aligned}
$$

Note that

$$
\begin{aligned}
\left(\sum_{i=1}^{n} \frac{q_{i}\left(x_{1}-x_{i}\right)}{x_{i}}+1\right)\left(\sum_{i=1}^{n} q_{i} x_{i}\right)-x_{1}^{2}\left(\sum_{i=1}^{n} \frac{q_{i}}{x_{i}}\right) \\
=\left(\sum_{i=1}^{n} \frac{q_{i}\left(x_{1}-x_{i}\right)}{x_{i}}\right)\left(\sum_{i=1}^{n} q_{i} x_{i}\right)-\sum_{i=1}^{n} \frac{q_{i}\left(x_{1}^{2}-x_{i}^{2}\right)}{x_{i}} \\
=\sum_{i=1}^{n} q_{i} \frac{\left(x_{1}-x_{i}\right)}{x_{i}}\left(\left(q_{1}-1\right) x_{1}+\sum_{j \neq 1, i} q_{j} x_{j}+\left(q_{i}-1\right) x_{i}\right) .
\end{aligned}
$$

Hence,

$$
g(\mathbf{x} ; \mathbf{q})=\frac{\sum_{i=1}^{n} q_{i}\left(\left(x_{1}-x_{i}\right) x_{n} / x_{i}\right)\left(\left(q_{1}-1\right)+\sum_{j \neq 1, i}\left(q_{j} x_{j} / x_{1}\right)+\left(q_{i}-1\right) x_{i} / x_{1}\right)}{\left(\sum_{i=1}^{n} q_{i} x_{i} / x_{n}\right)\left(\sum_{i=1}^{n} q_{i} x_{1} / x_{i}\right)} \leq 0 .
$$

Since $x / y>(x+t) /(y+t)$ when $x>y>0, t>0$, it follows from the above expression that $g(\mathbf{x} ; \mathbf{q}) \leq g\left(\mathbf{x}_{t} ; \mathbf{q}\right)$. The conditions for equality can be checked easily and this completes the proof.

LeMma 2.5. For $0<q<1$, when $0<y<x \leq 1$,

$$
f(q)=2 q\left(x^{1 / q}-y^{1 / q}\right)
$$

is an increasing function of $q$. When $1 \leq y<x, f(q)$ is a decreasing function of $q$.

Proof. We have

$$
f^{\prime}(q)=2\left(x^{1 / q}-y^{1 / q}\right)-2\left(\ln \left(x^{1 / q}\right) x^{1 / q}-\ln \left(y^{1 / q}\right) y^{1 / q}\right) .
$$

So it is enough to show that $u-u \ln u$ increases for $0<u \leq 1$ and decreases for $u \geq 1$ and this is easy to check.

Lemma 2.6. For $0<q<1,(1-q)^{1 / q-1}$ is an increasing function of $q$, in particular,

$$
(1-q)^{1 / q-1} \leq \frac{1}{2}
$$

when $0<q \leq 1 / 2$ and the above inequality reverses when $1 / 2 \leq q<1$. In either case, equality holds if and only if $q=1 / 2$. 
Proof. It suffices to show that $f^{\prime}(q)>0$ for $0<q<1$ with $f(q)=(1 / q-1) \ln (1-q)$. Now $f^{\prime}(q)=-h(q) / q^{2}$ with $h(q)=q+\ln (1-q)<0$ for $0<q<1$ and this completes the proof.

The following lemma is due to Wu et al. [25] (see also [2, pages 317-318]).

Lemma 2.7. Let $2 \leq r \leq n, \mathbf{x}=\left(x_{1}, \ldots, x_{n}\right), x_{1} \leq x_{2} \leq \cdots \leq x_{n}$. There exists $\mathbf{y}=\left(y_{1}, \ldots, y_{r}\right)$ with $x_{1} \leq y_{1} \leq \cdots \leq y_{r} \leq x_{n}$ such that $P_{n, i}(\mathbf{x})=P_{r, i}(\mathbf{y}), 0 \leq i \leq r$. Moreover, if $x_{1}, \ldots, x_{n}$ are not all equal, then $y_{1}, \ldots, y_{r}$ are also not all equal.

\section{Some monotonicity properties}

Theorem 3.1. Let $r>s, t \geq 0$.

(i) If $\Delta_{r, s, t, \alpha} \leq 1$, then $\Delta_{r, s, t, \beta} \leq 1$ for $\beta \leq \alpha$. If $\Delta_{r, s, t, \alpha} \geq 1$, then $\Delta_{r, s, t, \beta} \geq 1$ for $\beta \geq \alpha$.

(ii) Let $\alpha \leq 1$. If $\Delta_{r, s, t} \leq x_{n} /\left(t+x_{n}\right)$, then $\Delta_{r, s, t, \alpha} \leq\left(x_{n} /\left(t+x_{n}\right)\right)^{2-\alpha}$. If $\Delta_{r, s, t} \geq x_{1} /\left(t+x_{1}\right)$, then $\Delta_{r, s, t, \alpha} \geq\left(x_{1} /\left(t+x_{1}\right)\right)^{2-\alpha}$.

(iii) $\Delta_{r, s, t, \alpha} \leq 1$ for $\alpha \leq 0$ and for any $s \neq 1, \alpha \leq 1, \Delta_{1, s, t, \alpha} \leq 1$.

(iv) For any $r \neq 1, \min \left(\left(\left(t+x_{n}\right) / x_{n}\right)^{r-2},\left(\left(t+x_{1}\right) / x_{1}\right)^{r-2}\right) \leq \Delta_{r, 1, t, r} \leq \max (((t+$ $\left.\left.\left.x_{n}\right) / x_{n}\right)^{r-2},\left(\left(t+x_{1}\right) / x_{1}\right)^{r-2}\right)$.

(v) For $-1 \leq s \neq 1 \leq 2$,

$$
\frac{x_{n}}{t+x_{n}} \geq \Delta_{1, s, t} \geq \frac{x_{1}}{t+x_{1}}
$$

with equality holding if and only if $t=0$ or $x_{1}=\cdots=x_{n}$.

Proof. (i) Let $f(t)=\left|M_{n, r, t}^{\alpha}-M_{n, s, t}^{\alpha}\right|$. Since $\mathbf{x}$ is arbitrary, $\Delta_{r, s, t, \alpha} \leq 1$ is then equivalent to $f^{\prime}(0) \leq 0$ or the second inequality below:

$$
\frac{M_{n, r}^{\beta-r}}{M_{n, s}^{\beta-s}} \leq \frac{M_{n, r}^{\alpha-r}}{M_{n, s}^{\alpha-s}} \leq \frac{M_{n, r-1}^{1-r}}{M_{n, s-1}^{1-s}}
$$

Now $\Delta_{r, s, t, \beta} \leq 1$ follows from the first inequality above. This proves the first assertion and the second assertion follows similarly.

(ii) We will prove the first assertion for $0<\alpha<1$ and the other proofs are similar. Let $f(t)=\left(t+x_{n}\right)^{2-\alpha}\left(M_{n, r, t}^{\alpha}-M_{n, s, t}^{\alpha}\right)$, then it suffices to show $f^{\prime}(0) \leq 0$, or equivalently,

$$
(2-\alpha)\left(M_{n, r}^{\alpha}-M_{n, s}^{\alpha}\right) \leq \alpha x_{n}\left(M_{n, s}^{\alpha-1}\left(\frac{M_{n, s}}{M_{n, s-1}}\right)^{1-s}-M_{n, r}^{\alpha-1}\left(\frac{M_{n, r}}{M_{n, r-1}}\right)^{1-r}\right) .
$$

We also have

$$
\frac{M_{n, s}^{1-\alpha}}{\alpha}\left(M_{n, r}^{\alpha}-M_{n, s}^{\alpha}\right) \leq M_{n, r}-M_{n, s} \leq x_{n}\left(\left(\frac{M_{n, s}}{M_{n, s-1}}\right)^{1-s}-\left(\frac{M_{n, r}}{M_{n, r-1}}\right)^{1-r}\right),
$$


where the first inequality above follows from the mean value theorem and the second inequality follows from $\Delta_{r, s, t} \leq x_{n} /\left(t+x_{n}\right)$. Similarly, by using the mean value theorem, we get

$$
\frac{M_{n, r}^{\alpha}-M_{n, s}^{\alpha}}{M_{n, s}^{\alpha-1}-M_{n, r}^{\alpha-1}} \leq \frac{\alpha}{1-\alpha} M_{n, r} \leq \frac{\alpha}{1-\alpha} x_{n}\left(\frac{M_{n, r}}{M_{n, r-1}}\right)^{1-r}
$$

where the last inequality follows from $M_{n, r}^{r}=\sum_{i=1}^{n} \omega_{i} x_{i}^{r} \leq \sum_{i=1}^{n} \omega_{i} x_{n} x_{i}^{r-1}=x_{n} M_{n, r-1}^{r-1}$. Now (ii) follows by rewriting (3.4) and (3.5) as

$$
\begin{aligned}
& M_{n, r}^{\alpha}-M_{n, s}^{\alpha} \leq \alpha M_{n, s}^{\alpha-1} x_{n}\left(\left(\frac{M_{n, s}}{M_{n, s-1}}\right)^{1-s}-\left(\frac{M_{n, r}}{M_{n, r-1}}\right)^{1-r}\right), \\
& (1-\alpha)\left(M_{n, r}^{\alpha}-M_{n, s}^{\alpha}\right) \leq \alpha x_{n}\left(M_{n, s}^{\alpha-1}-M_{n, r}^{\alpha-1}\right)\left(\frac{M_{n, r}}{M_{n, r-1}}\right)^{1-r}
\end{aligned}
$$

and adding the above two inequalities.

(iii) $\Delta_{1, s, t, \alpha} \leq 1$, for $s \neq 1, \alpha \leq 1$, follows from the result of Aczél and Páles [1] and (i). Again by (i), in order to show $\Delta_{r, s, t, \alpha} \leq 1$ for $\alpha \leq 0$, it suffices to show $\Delta_{r, s, t, 0} \leq 1$. Let $f(t)=\ln M_{n, r, t}-\ln M_{n, s, t}$, it then suffices to show $f^{\prime}(0) \leq 0$ or $R_{n, s} \leq R_{n, r}$, where $R_{n, r}:=$ $M_{n, r}^{r} / M_{n, r-1}^{r-1}$. The last inequality holds by a result of Beckenbach [6, Theorem 1].

(iv) This follows by applying Lemma 2.1 to $f(x)=(t+x)^{r}, g(x)=x^{r}, r \neq 0$ and $f(x)=$ $\ln (t+x), g(x)=\ln x$ when $r=0$.

(v) We will prove the left-hand side inequality of (3.1) and the other proofs are similar. For $0 \leq s<1$, let

$$
D_{n}(\mathbf{x}, t)=x_{n}\left(A_{n}-M_{n, s}\right)-\left(t+x_{n}\right)\left(A_{n, t}-M_{n, s, t}\right) .
$$

We want to show $D_{n} \geq 0$ here. We can assume $x_{1}<x_{2}<\cdots<x_{n}$ and prove by induction that the case $n=1$ is clear, so we will start with $n>1$ variables assuming the inequality holds for $n-1$ variables. Then

$$
\begin{aligned}
\frac{\partial D_{n}}{\partial x_{n}} & =\left(A_{n}-M_{n, s}\right)-\left(A_{n, t}-M_{n, s, t}\right)+\omega_{n}\left(\left(A_{n}-M_{n, s}^{1-s} x_{n}^{s}\right)-\left(A_{n, t}-M_{n, s, t}^{1-s}\left(t+x_{n}\right)^{s}\right)\right) \\
& \geq \omega_{n}\left(\left(A_{n}-M_{n, s}\right)-\left(A_{n, t}-M_{n, s, t}\right)+\left(A_{n}-M_{n, s}^{1-s} x_{n}^{s}\right)-\left(A_{n, t}-M_{n, s, t}^{1-s}\left(t+x_{n}\right)^{s}\right)\right) \\
& =\omega_{n}\left(M_{n, s, t}^{1-s}\left(t+x_{n}\right)^{s}+M_{n, s, t}-2 t-M_{n, s}-M_{n, s}^{1-s} x_{n}^{s}\right),
\end{aligned}
$$

where the inequality follows from $\Delta_{1, s, t} \leq 1$. Now we consider

$$
g(t)=M_{n, s, t}^{1-s}\left(t+x_{n}\right)^{s}+M_{n, s, t}-2 t
$$


3558 A new approach to Ky Fan-type inequalities

and we have

$$
\begin{aligned}
g^{\prime}(t) & =(1-s)\left(\frac{t+x_{n}}{M_{n, s, t}}\right)^{s}\left(\frac{M_{n, s, t}}{M_{n, s-1, t}}\right)^{1-s}+s\left(\frac{M_{n, s, t}}{t+x_{n}}\right)^{1-s}+\left(\frac{M_{n, s, t}}{M_{n, s-1, t}}\right)^{1-s}-2 \\
& \geq(1-s) y^{s}+s y^{s-1}-1 \geq y^{(1-s) s+s(s-1)}-1=0,
\end{aligned}
$$

where $y=\left(t+x_{n}\right) / M_{n, s, t} \geq 1$ and the first inequality above follows from $\left(M_{n, s, t} / M_{n, s-1, t}\right)^{1-s}$ $\geq 1$. The last inequality above follows from the arithmetic-geometric mean inequality. Thus $g^{\prime}(t) \geq 0$, hence $g(t) \geq g(0)=M_{n, s}+M_{n, s}^{1-s} x_{n}^{s}$ and it follows $\partial D_{n} / \partial x_{n} \geq 0$ and by letting $x_{n}$ tend to $x_{n-1}$, we have $D_{n} \geq D_{n-1}$ (with weights $\omega_{1}, \ldots, \omega_{n-2}, \omega_{n-1}+\omega_{n}$ ) and thus the right-hand side inequality of (3.1) holds by induction. It is easy to see that the equality holds if and only if $t=0$ or $x_{1}=\cdots=x_{n}$.

For $-1 \leq s<0$, we have

$$
\frac{1}{\omega_{1}} \frac{\partial D_{n}}{\partial x_{1}}=-t-x_{n}\left(\frac{M_{n, s}}{x_{1}}\right)^{1-s}+\left(t+x_{n}\right)\left(\frac{M_{n, s, t}}{t+x_{1}}\right)^{1-s}:=-t-f\left(x_{1}\right) .
$$

Consider

$$
f^{\prime}\left(x_{1}\right)=-(1-s) \sum_{j=2}^{n} \omega_{j}\left(\left(\frac{M_{n, s}}{x_{1}}\right)^{1-2 s} \cdot \frac{x_{n} x_{j}^{s}}{x_{1}^{s+1}}-\left(\frac{M_{n, s, t}}{t+x_{1}}\right)^{1-2 s} \frac{\left(t+x_{n}\right)\left(t+x_{j}\right)^{s}}{\left(t+x_{1}\right)^{s+1}}\right) \leq 0 .
$$

The last inequality holds, since when $-1 \leq s<0,2 \leq j \leq n$, we have

$$
\frac{x_{n}}{t+x_{n}} \cdot\left(\frac{x_{j}}{t+x_{j}}\right)^{s} \geq\left(\frac{x_{j}}{t+x_{j}}\right)^{1+s} \geq\left(\frac{x_{1}}{t+x_{1}}\right)^{1+s}
$$

and $x_{j} / x_{1} \geq\left(t+x_{j}\right) /\left(t+x_{1}\right)$ so that

$$
\left(\frac{M_{n, s}}{x_{1}}\right)^{1-2 s} \geq\left(\frac{M_{n, s, t}}{t+x_{1}}\right)^{1-2 s}
$$

Thus by a similar argument as above, we deduce that $f\left(x_{1}\right) \geq-t$ and $\partial D_{n} / \partial x_{1} \leq 0$, which further implies $D_{n} \geq 0$ with equality holding if and only if $t=0$ or $x_{1}=\cdots=x_{n}$.

For $1<s \leq 2$, it suffices to show $\partial D_{n} / \partial t \leq 0$ or, equivalently,

$$
\frac{M_{n, s}^{s-1}}{x_{n}} \leq \frac{\left(M_{n, s}^{s-1}-M_{n, s-1}^{s-1}\right)}{\left(M_{n, s}-A_{n}\right)} .
$$

The above inequality certainly holds for $s=2$, otherwise it follows from $M_{n, s}^{s-1} / x_{n} \leq x_{n}^{s-2}$ and Lemma 2.2 with $u=s-1, v=s, w=1$. 
We remark here that one cannot compare $M_{n, r, t}-M_{n, s, t}$ and $M_{n, r}-M_{n, s}$ in general. For example, let $f(t)=G_{n, t}-H_{n, t}$. Then $f^{\prime}(0)=G_{n} / H_{n}-H_{n}^{2} / M_{n,-2}^{2}$. By a change of variables $x_{i} \rightarrow 1 / x_{n-i+1}$, we can rewrite this as $f^{\prime}(0)=\left(A_{n}^{3}-G_{n} M_{n, 2}^{2}\right) /\left(A_{n}^{2} G_{n}\right)$ and by considering the case $n=2$ with $q_{1}=q_{2}=1 / 2, x_{2}=1$, it is easy to see that $f^{\prime}(0)<0$ if $x_{1}=1 / 2$ and $f^{\prime}(0)>0$ if $x_{1}=0$.

Corollary 3.2. Inequality (1.2) holds for $r=1,-1 \leq s<1$ and $1<r \leq 2, s=1$.

Proof. This follows from Theorem 3.1 (v) and that (1.2) and (1.4) are equivalent.

The above result was first proved by the author in [14, Theorem 3.2]; in fact it was shown there that those are the only cases (1.2) can hold for $r=1$ or $s=1$. Thus by noticing the equivalence of (1.2) and (1.4), we obtain the following.

Corollary 3.3. Inequality (3.1) holds for all $t \geq 0$ if and only if $-1 \leq s \neq 1 \leq 2$.

Corollary 3.4. For $-1 \leq s<1$,

$$
\frac{x_{n}}{M_{n, s-1}^{1-s}} \geq \frac{\left(A_{n}-M_{n, s}\right)}{\left(M_{n, s}^{1-s}-M_{n, s-1}^{1-s}\right)} \geq \frac{x_{1}}{M_{n, s-1}^{1-s}} .
$$

Proof. Theorem 3.1(v) implies $f(t)=\left(t+x_{n}\right)\left(A_{n, t}-M_{n, s, t}\right)$ is a decreasing function of $t$ and $f^{\prime}(0) \leq 0$ implies the left-hand side inequality of (3.16). The proof of the right-hand side inequality of (3.16) is similar.

By a change of variables $x_{i} \rightarrow 1 / x_{n-i+1}$, the left-hand side inequality of (3.16) when $s=-1$ gives

$$
A_{n}-H_{n} \leq \frac{H_{n}}{x_{1} A_{n}} \sigma_{n}
$$

a refinement of the left-hand side inequality of (1.2) for $r=1, s=-1$.

\section{Some equivalent inequalities}

THEOREM 4.1. The following inequalities are equivalent:

(i) $A_{n}-G_{n} \geq \sigma_{n} / 2 x_{n}$;

(ii) $A_{n}-G_{n} \leq \sigma_{n} / 2 x_{1}$;

(iii) $A_{n}-G_{n} \leq\left(x_{n} / H_{n}\right)\left(G_{n}-H_{n}\right)$;

(iv) $A_{n}-G_{n} \geq\left(x_{1} / H_{n}\right)\left(G_{n}-H_{n}\right)$;

(v) $G_{n}-H_{n} \geq H_{n} \sigma_{n} / 2 x_{n}^{2}$;

(vi) $G_{n}-H_{n} \leq H_{n} \sigma_{n} / 2 x_{1}^{2}$.

In particular, inequalities (i)-(vi) are all valid since (i) holds by Corollary 3.2.

Proof. We first show (ii) $\Rightarrow$ (iii) $\Rightarrow$ (i) and similarly one can show (i) $\Rightarrow$ (iv) $\Rightarrow$ (ii).

(iii) $\Rightarrow$ (i). Let $f(t)=\left(x_{n}+t\right)\left(A_{n, t}-G_{n, t}\right), t \geq 0$. It is easy to see that $\lim _{t \rightarrow \infty} f(t)=\sigma_{n} / 2$. Thus it suffices to show $f(t)$ is a decreasing function of $t$ in order to prove (i). Since $\mathbf{x}$ is arbitrary, it suffices to have $f^{\prime}(0)=A_{n}-G_{n}+x_{n}\left(1-G_{n} / H_{n}\right) \leq 0$, which is just (iii).

(ii) $\Rightarrow$ (iii). Let $f(t)=A_{n, t}-G_{n, t}+\left(x_{n}+t\right)\left(1-G_{n, t} / H_{n, t}\right), t \geq 0$. It is easy to see that $\lim _{t \rightarrow \infty} f(t)=0$, so it suffices to show $f^{\prime}(t) \geq 0$ in order to prove (iii). Since $\mathbf{x}$ is arbitrary, 
it suffices to show $f^{\prime}(0) \geq 0$. Calculation yields

$$
\frac{f^{\prime}(0)}{G_{n}}=2\left(\frac{1}{G_{n}}-\frac{1}{H_{n}}\right)+x_{n}\left(\frac{1}{M_{n,-2}^{2}}-\frac{1}{H_{n}^{2}}\right) .
$$

By a change of variables $x_{i} \rightarrow 1 / x_{n-i+1}$, the right-hand side inequality of (4.1) becomes

$$
2\left(G_{n}-A_{n}\right)+\frac{1}{x_{1}} \sigma_{n} \geq 0
$$

by (ii).

Now we show that (i) and (v) are equivalent; similarly one can show that (i) and (vi) are equivalent.

(i) $\Rightarrow(v)$. We have shown that (i) and (iii) are equivalent, and hence (v) follows.

(v) $\Rightarrow(\mathrm{i})$. Let $f(t)=A_{n, t}-G_{n, t}-\sigma_{n} / 2\left(x_{n}+t\right)$. It is easy to see that $\lim _{t \rightarrow \infty} f(t)=0$, so it suffices to show $f^{\prime}(t) \leq 0$ in order to prove (i). Since $\mathbf{x}$ is arbitrary, it suffices to show $f^{\prime}(0) \leq 0$, which is just $(\mathrm{v})$.

Theorem 4.2. The following inequalities are equivalent:

(i) $A_{n}-H_{n} \geq\left(H_{n} / x_{n} A_{n}\right) \sigma_{n}$;

(ii) $A_{n}-H_{n} \leq \sigma_{n} / x_{1}$.

In particular, $A_{n}-H_{n} \geq \sigma_{n} / x_{n}$ implies $A_{n}-H_{n} \leq \sigma_{n} / x_{1}$. Moreover, it also holds that

$$
A_{n}-H_{n} \geq \frac{M_{n, 2}^{2}-2 A_{n} H_{n}+H_{n}^{2}}{x_{n}}
$$

with equality holding if and only if $x_{1}=\cdots=x_{n}$, which implies (3.17), and (3.17) further implies $A_{n}-H_{n} \leq \sigma_{n} / x_{1}$.

Proof. We first show that inequality (i) is equivalent to (ii). Let $f(t)=\left(x_{1}+t\right)\left(A_{n, t}-\right.$ $\left.H_{n, t}\right), g(t)=\left(x_{n}+t\right) A_{n, t}\left(A_{n, t}-H_{n, t}\right) / H_{n, t}$.

(i) $\Rightarrow$ (ii). By using similar arguments as in the proof of Theorem 4.1, (ii) holds if $f^{\prime}(0)$ $\geq 0$; by a change of variables $x_{i} \rightarrow 1 / x_{n-i+1}$, one checks that $f^{\prime}(0) \geq 0$ is equivalent to (i).

(ii) $\Rightarrow$ (i). Similarly, (i) holds if $g^{\prime}(0) \leq 0$. By a change of variables $x_{i} \rightarrow 1 / x_{n-i+1}$, one checks that $g^{\prime}(0) \leq 0$ is equivalent to

$$
A_{n}-H_{n} \leq \frac{M_{n, 2}^{2}-2 A_{n} H_{n}+H_{n}^{2}}{x_{1}}=\frac{\sigma_{n}}{x_{1}}+\frac{\left(A_{n}-H_{n}\right)^{2}}{x_{1}} .
$$

Thus (ii) implies (4.4), hence (i).

Similarly, one can show that (4.3) implies (3.17), and hence $A_{n}-H_{n} \leq \sigma_{n} / x_{1}$. It now remains to show (4.3). In fact, both (4.3) and (3.17) follow from the following identity:

$$
A_{n}-H_{n}=\sum_{i=1}^{n} \frac{\omega_{i}\left(x_{i}-H_{n}\right)^{2}}{x_{i}}=\frac{H_{n}}{A_{n}} \sum_{i=1}^{n} \frac{\omega_{i}\left(x_{i}-A_{n}\right)^{2}}{x_{i}} .
$$


We note here that it follows from (4.5) that for $t \geq 0$,

$$
\frac{1}{H_{n, t}}-\frac{1}{A_{n, t}}=\frac{1}{A_{n, t}^{2}} \sum_{i=1}^{n} \frac{\omega_{i}\left(x_{i}-A_{n}\right)^{2}}{x_{i, t}}
$$

Upon integration of both sides above from 0 to $\infty$, we obtain an identity of Dinghas [12, (9.9)]:

$$
\begin{aligned}
\ln A_{n}-\ln G_{n} & =\left.\left(\ln G_{n, t}-\ln A_{n, t}\right)\right|_{0} ^{\infty} \\
& =\int_{0}^{\infty}\left(\frac{1}{H_{n, t}}-\frac{1}{A_{n, t}}\right) d t \\
& =\int_{0}^{\infty}\left(\frac{1}{A_{n, t}^{2}} \sum_{i=1}^{n} \frac{\omega_{i}\left(x_{i}-A_{n}\right)^{2}}{x_{i}+t}\right) d t \\
& =\left.\left(-\sum_{i=1}^{n} \omega_{i}\left(x_{i}-A_{n}\right)^{2} J\left(x_{i}+t, A_{n, t}\right)\right)\right|_{0} ^{\infty} \\
& =\sum_{i=1}^{n} \omega_{i}\left(x_{i}-A_{n}\right)^{2} J\left(x_{i}, A_{n}\right),
\end{aligned}
$$

where, as given in $[12,(9.8)]$,

$$
J(x, y)=\int_{0}^{\infty} \frac{\alpha d \alpha}{(1+\alpha)(x+\alpha y)^{2}} .
$$

We note here that (4.5) also improves Mercer's proposition [20, Proposition 4], as one checks directly. To end this section, we give a refinement of (1.2) based on the results in [20].

Theorem 4.3. The following inequality

$$
\sum_{i=1}^{n} \frac{\omega_{i}\left(x_{i}-G_{n}\right)^{2}}{x_{i}+\min \left(x_{i}, G_{n}\right)} \geq A_{n}-G_{n} \geq \sum_{i=1}^{n} \frac{\omega_{i}\left(x_{i}-A_{n}\right)^{2}}{x_{i}+\max \left(x_{i}, A_{n}\right)}
$$

holds with equality holding if and only if $x_{1}=\cdots=x_{n}$.

Proof. The second inequality is [20, Proposition 1] and the first inequality follows from

$$
A_{n}-G_{n}=\exp \left(\ln A_{n}\right)-\exp \left(\ln G_{n}\right) \leq A_{n}\left(\ln A_{n}-\ln G_{n}\right) \leq \sum_{i=1}^{n} \frac{\omega_{i}\left(x_{i}-A_{n}\right)^{2}}{x_{i}+\min \left(x_{i}, A_{n}\right)}
$$

where the first inequality above follows from the mean value theorem and the second inequality follows from [20, Proposition 2]. 
3562 A new approach to Ky Fan-type inequalities

\section{A sharpening of Sierpiński's inequality}

Theorem 5.1. For $t \geq 0, q=\min \left\{\omega_{i}\right\}>0$,

$$
\begin{aligned}
& \left(\frac{x_{n}}{x_{n}+t}\right)^{2} \geq \frac{(1-q) \ln A_{n, t}+q \ln H_{n, t}-\ln G_{n, t}}{(1-q) \ln A_{n}+q \ln H_{n}-\ln G_{n}} \geq\left(\frac{x_{1}}{x_{1}+t}\right)^{2}, \\
& \left(\frac{x_{n}}{x_{n}+t}\right)^{2} \geq \frac{\ln G_{n, t}-q \ln A_{n, t}-(1-q) \ln H_{n, t}}{\ln G_{n}-q \ln A_{n}-(1-q) \ln H_{n}} \geq\left(\frac{x_{1}}{x_{1}+t}\right)^{2}
\end{aligned}
$$

with equality holding if and only if $t=0, q=1 / 2$, or $x_{1}=\cdots=x_{n}$.

Proof. The proof uses the ideas in [5]. We will prove the left-hand side inequality of (5.1) and the proofs for other inequalities are similar, we only point out that one needs Lemma 2.4 in order to prove (5.2). We may assume $t>0$ to be fixed and define

$$
\begin{aligned}
f_{n}\left(\mathbf{x}_{n}, q\right)= & x_{n}^{2}\left((1-q) \ln A_{n}+q \ln H_{n}-\ln G_{n}\right) \\
& -\left(x_{n}+t\right)^{2}\left((1-q) \ln A_{n, t}+q \ln H_{n, t}-\ln G_{n, t}\right),
\end{aligned}
$$

where we regard $A_{n}, G_{n}, H_{n}, A_{n, t}, G_{n, t}, H_{n, t}$ as functions of $\mathbf{x}_{n}=\left(x_{1}, \ldots, x_{n}\right)$. Then

$$
\begin{aligned}
g_{n}\left(x_{2}, \ldots, x_{n-1}\right): & =\frac{1}{\omega_{1}} \frac{\partial f_{n}}{\partial x_{1}} \\
& =x_{n}^{2}\left(\frac{1-q}{A_{n}}+\frac{q H_{n}}{x_{1}^{2}}-\frac{1}{x_{1}}\right)-\left(x_{n}+t\right)^{2}\left(\frac{1-q}{A_{n, t}}+\frac{q H_{n, t}}{\left(x_{1}+t\right)^{2}}-\frac{1}{x_{1}+t}\right) .
\end{aligned}
$$

By setting $\omega_{i} / q=q_{i}$, it follows from Lemma 2.3 that $g_{n}=\left(1 / \omega_{1}\right)\left(\partial f_{n} / \partial x_{1}\right) \leq 0$ with equality holding if and only if $n=1$ or $n=2, q=1 / 2$. By letting $x_{1}$ tend to $x_{2}$, we have

$$
f_{n}\left(\mathbf{x}_{n}, q\right) \geq f_{n-1}\left(\mathbf{x}_{n-1}, q\right) \geq f_{n-1}\left(\mathbf{x}_{n-1}, q^{\prime}\right)
$$

where $\mathbf{x}_{n-1}=\left(x_{2}, \ldots, x_{n}\right)$ with weights $\omega_{1}+\omega_{2}, \ldots, \omega_{n-1}, \omega_{n}$ and $q^{\prime}=\min \left(\omega_{1}+\omega_{2}, \ldots, \omega_{n}\right)$. Here the second inequality above follows from $\Delta_{1,-1, t, 0} \leq\left(x_{n} /\left(t+x_{n}\right)\right)^{2}$, which is a consequence of Theorem 3.1 (v) and (ii).

It then follows by induction that $f_{n} \geq f_{n-1} \geq \cdots \geq f_{2}=0$ when $q=1 / 2$ in $f_{2}$ or else $f_{n} \geq f_{n-1} \geq \cdots \geq f_{1}=0$ and this completes the proof.

By letting $t \rightarrow \infty$ in (5.1) and (5.2), we recover the following result of the author [14, Theorem 5.1], which can be regarded as a sharpening of Sierpiński's inequality [23] for the weighted cases. 
Corollary 5.2. For $q=\min \left\{\omega_{i}\right\}>0$,

$$
\begin{aligned}
& \frac{1-2 q}{2 x_{1}^{2}} \sigma_{n} \geq(1-q) \ln A_{n}+q \ln H_{n}-\ln G_{n} \geq \frac{1-2 q}{2 x_{n}^{2}} \sigma_{n}, \\
& \frac{1-2 q}{2 x_{1}^{2}} \sigma_{n} \geq \ln G_{n}-q \ln A_{n}-(1-q) \ln H_{n} \geq \frac{1-2 q}{2 x_{n}^{2}} \sigma_{n}
\end{aligned}
$$

with equality holding if and only if $q=1 / 2$ or $x_{1}=\cdots=x_{n}$.

Corollary 5.3. For $q=\min \left\{\omega_{i}\right\}>0$,

$$
q A_{n} H_{n}+(1-q) M_{n, 2}^{2} \geq A_{n}^{2} \geq(1-q) A_{n} H_{n}+q M_{n, 2}^{2}
$$

with equality holding if and only if $q=1 / 2$ or $x_{1}=\cdots=x_{n}$.

Proof. We will prove the right-hand side inequality of (5.8) and the other proof is similar. Theorem 5.1 implies that $f(t)=\left(t+x_{n}\right)^{2}\left((1-q) \ln A_{n, t}+q \ln H_{n, t}-\ln G_{n, t}\right)$ is a decreasing function of $t$ and $f^{\prime}(0) \leq 0$ gives

$$
x_{n}^{2}\left(\frac{1-q}{A_{n}}+\frac{q H_{n}}{M_{n,-2}^{2}}-\frac{1}{H_{n}}\right)+2 x_{n}\left((1-q) \ln A_{n}+q \ln H_{n}-\ln G_{n}\right) \leq 0 .
$$

Since $(1-q) \ln A_{n}+q \ln H_{n}-\ln G_{n} \geq 0$ by Corollary 5.2, we must have $(1-q) / A_{n}+$ $q H_{n} / M_{n,-2}^{2}-1 / H_{n} \leq 0$ and (5.8) follows by a change of variables $x_{i} \rightarrow 1 / x_{n-i+1}$.

Corollary 5.4. For $q=\min \left\{\omega_{i}\right\}, t \geq 0, \Delta_{1,0, t, \alpha} \leq 1$ for $\alpha \leq(1-q)^{-1}$ and $\Delta_{1,0, t, \alpha} \geq 1$ for $\alpha \geq q^{-1}$.

Proof. We will show the first assertion and the proof for the other one is similar. By Theorem 3.1(i), it suffices to prove the above result for $\alpha_{0}=(1-q)^{-1}$. Let $f(t)=A_{n, t}^{\alpha_{0}}-$ $G_{n, t}^{\alpha_{0}}$; by similar argument as in the proof of Theorem 3.1(i), it suffices to show $f^{\prime}(0) \leq 0$, which is equivalent to $A_{n}^{q} H_{n}^{1-q} \leq G_{n}$, and this last inequality follows from (5.7) and this completes the proof.

\section{Some refinements of Ky Fan-type inequalities}

Theorem 1.2 and Corollary 5.4 motivate the following two results.

Theorem 6.1. For $0<q \leq \min \left\{\omega_{i}\right\}$,

$$
x_{1}^{1 /(1-q)-2} \sigma_{n} \geq 2(1-q)\left(A_{n}^{1 /(1-q)}-G_{n}^{1 /(1-q)}\right) \geq x_{n}^{1 /(1-q)-2} \sigma_{n}
$$

with equality holding if and only if $n=2, q=1 / 2$, or $x_{1}=x_{2}=\cdots=x_{n}$.

Proof. We prove the right-hand side inequality of (6.1) first. By homogeneity, we may assume $0 \leq x_{1} \leq x_{2} \leq \cdots \leq x_{n}=1$ here and define

$$
D_{n}\left(x_{1}, \ldots, x_{n-1}\right)=2(1-q)\left(A_{n}^{1 /(1-q)}-G_{n}^{1 /(1-q)}\right)-\sigma_{n} .
$$


We want to show $D_{n} \geq 0$. Let $\mathbf{a}=\left(a_{1}, \ldots, a_{n-1}\right) \in[0,1]^{n-1}$ be the point in which the absolute minimum of $D_{n}$ is reached.

We may assume $a_{1} \leq a_{2} \leq \cdots \leq a_{n-1}$ and let $a_{n}=1$. If $a_{i}=a_{i+1}$ for some $1 \leq i \leq n-1$, by combining $a_{i}$ with $a_{i+1}$ and $\omega_{i}$ with $\omega_{i+1}$, while noticing that increasing $q$ will decrease the value of $(1-q)\left(A_{n}^{1 /(1-q)}-G_{n}^{1 /(1-q)}\right)$ by Lemma 2.5 , we can reduce the determination of the absolute minimum of $D_{n}$ to that of $D_{n-1}$ with different weights. Thus without loss of generality, we may assume $a_{1}<a_{2}<\cdots<a_{n-1}<1$. If $a_{1}>0$, then $\mathbf{a}$ is an interior point of $[0,1]^{n-1}$, and we obtain

$$
\nabla D_{n}\left(a_{1}, \ldots, a_{n-1}\right)=0
$$

such that $a_{1}, \ldots, a_{n-1}$ solve the equation

$$
x^{2}-\left(A_{n}+A_{n}^{q /(1-q)}\right) x+G_{n}^{1 /(1-q)}=0 .
$$

The above equation has at most two roots (regarding $A_{n}, G_{n}$ as constants), so we are reduced to the case $n=3$. But if $a_{1}<a_{2}<1$ both satisfy (6.4), we will have

$$
a_{1} a_{2}=G_{3}^{1 /(1-q)}=a_{1}^{\omega_{1} /(1-q)} a_{2}^{\omega_{2} /(1-q)}
$$

which is impossible since $\omega_{1}+q \leq 1, \omega_{2}+q \leq 1$ and the two equalities cannot hold at the same time. Thus if $a_{1}>0$, we only need to prove $D_{2} \geq 0$. In this case, by letting $x=a_{1}>0$, we get

$$
D_{2}(x)=2(1-q)\left(\left(\omega_{1} x+\omega_{2}\right)^{1 /(1-q)}-x^{\omega_{1} /(1-q)}\right)-\omega_{1} \omega_{2}(x-1)^{2} .
$$

It is easy to check $D_{2}(1)=D_{2}^{\prime}(1)=0$ and

$$
\begin{aligned}
\frac{D^{\prime \prime}(x)}{2 \omega_{1}} & =\frac{q \omega_{1}}{1-q}\left(\omega_{1} x+\omega_{2}\right)^{(2 q-1) /(1-q)}-\left(\frac{\omega_{1}}{1-q}-1\right) x^{\omega_{1} /(1-q)-2}-\omega_{2} \\
& \geq \frac{q \omega_{1}}{1-q}+1-\frac{\omega_{1}}{1-q}-\omega_{2}=0
\end{aligned}
$$

with equality holding if and only if $x=1$ or $q=1 / 2$. Hence, by the Taylor expansion at 1 , $D_{2}(x) \geq 0$ with equality holding if and only if $x=1$ or $q=1 / 2$.

If $\mathbf{a}$ is a boundary point of $[0,1]^{n-1}$, then $a_{1}=0$ and (6.2) is reduced to

$$
E_{n}\left(x_{1}=0, \ldots, x_{n-1}\right)=2(1-q) A_{n}^{1 /(1-q)}-\sigma_{n} .
$$

We now show $E_{n} \geq 0$. Let $\left(a_{2}, \ldots, a_{n-1}\right) \in[0,1]^{n-2}$ be the point in which the absolute minimum of $E_{n}$ is reached. Similar to the argument above, we may assume $0=a_{1}<a_{2}<$ $\cdots<a_{n-1}<1$ and it is easy to show by using the method above that we only need to consider the case $n=3$. When $n=3$ and $0<a_{2}<1$, we have

$$
\frac{E_{3}^{\prime}\left(a_{2}\right)}{2 \omega_{2}}=A_{3}^{q /(1-q)}-\left(a_{2}-A_{3}\right)=0 .
$$


Using this, we get

$$
\begin{aligned}
\frac{(1-q) A_{3}}{2 \omega_{2}} E_{3}^{\prime \prime}\left(a_{2}\right) & =q \omega_{2} A_{3}^{q /(1-q)}-(1-q)\left(1-\omega_{2}\right) A_{3} \\
& =q \omega_{2}\left(a_{2}-A_{3}\right)-(1-q)\left(1-\omega_{2}\right) A_{3} \\
& =q \omega_{2}\left(\left(1-\omega_{2}\right) a_{2}-\omega_{3}\right)-(1-q)\left(1-\omega_{2}\right)\left(\omega_{2} a_{2}+\omega_{3}\right) \\
& =\omega_{2}\left(1-\omega_{2}\right)(2 q-1) a_{2}-q \omega_{2} \omega_{3}-(1-q)\left(1-\omega_{2}\right) \omega_{3}<0 .
\end{aligned}
$$

This implies that $E_{3}(x)$ takes its local maximum at $a_{2}$, so in order to show $E_{3} \geq 0$, we only need to show it for the cases $a_{2}=0$ or $a_{2}=1$ and we are then back to the case $n=2$. In this case, $E_{2} \geq 0$ is equivalent to $q^{1 /(1-q)} \geq q / 2$ and $g(q)=(1-q)^{1 /(1-q)}-q / 2 \geq 0$. The first inequality follows from Lemma 2.6 and one checks that $g(q)$ is a decreasing function of $q$, hence $g(q) \geq g(1 / 2)=0$. This now completes the proof for the right-hand side inequality of (6.1).

For the left-hand side inequality of (6.1), we may assume $1=x_{1} \leq x_{2} \leq \cdots \leq x_{n}$ and define

$$
F_{n}\left(x_{2}, \ldots, x_{n}\right)=\sigma_{n}-2(1-q)\left(A_{n}^{1 /(1-q)}-G_{n}^{1 /(1-q)}\right) .
$$

We want to show $F_{n} \geq 0$. Let $\mathbf{a}=\left(a_{2}, \ldots, a_{n}\right) \in[1, \infty)^{n-1}$ be the point in which the absolute minimum of $F_{n}$ is reached.

Again by Lemma 2.5 we may assume $a_{1}=1<a_{2}<a_{3}<\cdots<a_{n}$. If $a_{n}<\infty$, then a is an interior point of $[1, \infty)^{n-1}$, and we obtain

$$
\nabla F_{n}\left(a_{2}, \ldots, a_{n}\right)=0
$$

such that $a_{2}, \ldots, a_{n}$ solve (6.4), which has at most two roots (regarding $A_{n}, G_{n}$ as constants), so we are reduced to the case $n=3$. But if $1<a_{2}<a_{3}$, then we will have (6.5), which is again impossible.

Thus we only need to consider the case $n=2$ and $F_{2} \geq 0$ can be proved similarly to our treatment of $D_{2} \geq 0$. One checks easily here $F_{2}\left(x_{2}\right)=0$ if and only if $x_{2}=1$ or $q=1 / 2$.

So now we only need to consider the case $a_{n} \rightarrow \infty$. In this case, it is easy to see that if $q=1 / 2$, then $n=2$ and $F_{2}=0$. If $q<1 / 2$, then $1 /(1-q)<2$, hence

$$
\lim _{a_{n} \rightarrow \infty} F_{n}=\lim _{a_{n} \rightarrow \infty} a_{n}^{2} \cdot \lim _{a_{n} \rightarrow \infty} \frac{\sigma_{n}}{a_{n}^{2}}>0 .
$$

This now completes the proof for the left-hand side inequality of (6.1) with the conditions for equality readily checked.

Theorem 6.2. For $0<q \leq \min \left\{\omega_{i}\right\}$,

$$
x_{n}^{1 / q-2} \sigma_{n} \geq 2 q\left(A_{n}^{1 / q}-G_{n}^{1 / q}\right) \geq x_{1}^{1 / q-2} \sigma_{n}
$$

with equality holding if and only if $n=2, q=1 / 2$, or $x_{1}=x_{2}=\cdots=x_{n}$. 
Proof. We prove the left-hand side inequality first. By homogeneity, we may assume $0 \leq$ $x_{1} \leq x_{2} \leq \cdots \leq x_{n}=1$ here and define

$$
D_{n}\left(x_{1}, \ldots, x_{n-1}\right)=\sigma_{n}-2 q\left(A_{n}^{1 / q}-G_{n}^{1 / q}\right) .
$$

We want to show $D_{n} \geq 0$. Let $\mathbf{a}=\left(a_{1}, \ldots, a_{n-1}\right) \in[0,1]^{n-1}$ be the point in which the absolute minimum of $D_{n}$ is reached. As in the proof of Theorem 6.1 and again using Lemma 2.5, we may assume $a_{1}<a_{2}<\cdots<a_{n-1}<a_{n}=1$. If $a_{1}>0$, then $\mathbf{a}$ is an interior point of $[0,1]^{n-1}$, and we obtain

$$
\nabla D_{n}\left(a_{1}, \ldots, a_{n-1}\right)=0
$$

such that $a_{1}, \ldots, a_{n-1}$ solve the equation

$$
x^{2}-\left(A_{n}+A_{n}^{(1-q) / q}\right) x+G_{n}^{1 / q}=0 .
$$

The above equation has at most two roots (regarding $A_{n}, G_{n}$ as constants), so we are reduced to the case $n=3$. But if $a_{1}<a_{2}<1$ both satisfy (6.17), we will have

$$
a_{1} a_{2}=G_{3}^{1 / q}=a_{1}^{\omega_{1} / q} a_{2}^{\omega_{2} / q}
$$

which is impossible since $\omega_{1} \geq q, \omega_{2} \geq q$ and the two equalities cannot hold at the same time. Thus if $a_{1}>0$, we only need to prove $D_{2} \geq 0$. In this case, set $x=a_{1}>0$ and consider first the case $\omega_{1}=1-q, \omega_{2}=q$. Define

$$
g(u):=u+\frac{G_{2}^{1 / q}}{u} .
$$

We first observe that by (6.17),

$$
g(x)=A_{2}^{(1-q) / q}+A_{2}
$$

Note for $u \geq x, q \leq 1 / 3$,

$$
g^{\prime}(u)=1-\frac{G_{2}^{1 / q}}{u^{2}} \geq 1-\frac{G_{2}^{1 / q}}{x^{2}} \geq 0,
$$

since $0<x<1$ and $G_{2}=x^{1-q}$. As $x<A_{2}$ in our case, we then have $g(x) \leq g\left(A_{2}\right)=A_{2}+$ $G_{2}^{1 / q} / A_{2}$, a contradiction.

Now suppose $q>1 / 3$, then

$$
\begin{aligned}
D_{2}^{\prime \prime}(x) & =\frac{2(1-q)}{q}\left(q^{2}-(1-q)^{2} A_{2}^{(1-2 q) / q}+(1-2 q) x^{(1-3 q) / q}\right) \\
& \geq \frac{2(1-q)}{q}\left(q^{2}-(1-q)^{2}+(1-2 q)\right)=0,
\end{aligned}
$$

with equality holding if and only if $q=1 / 2$. As $D_{2}(1)=D_{2}^{\prime}(1)=0$, this shows $D_{2}(x) \geq 0$ by considering the Taylor expansion of $D_{2}$ at 1 . 
Now consider the case $\omega_{1}=q, \omega_{2}=1-q$, then $D_{2}^{\prime \prime}(x)=2 q(1-q)\left(1-A_{2}^{(1-2 q) / q}\right) \geq 0$ with equality holding if and only if $q=1 / 2$. As we also have $D_{2}(1)=D_{2}^{\prime}(1)=0$, this shows $D_{2}(x) \geq 0$.

Finally, we consider the case when $D_{n}$ reaches its absolute minimum at a with $a_{1}=0$. Define

$$
E_{n}\left(x_{1}=0, \ldots, x_{n-1}\right)=\sigma_{n}-2 q A_{n}^{1 / q} .
$$

We show now $E_{n} \geq 0$. Let $\mathbf{a}=\left(a_{2}, \ldots, a_{n-1}\right) \in[0,1]^{n-2}, 0<a_{2}<\cdots<a_{n-1}<1$, be the point in which the absolute minimum of $E_{n}$ is reached. Similar to the argument in the proof of Theorem 6.1 , we may assume $0=a_{1}<a_{2}<\cdots<a_{n-1}<1$ and it is easy to show by using the method there that we only need to consider the case $n=3$. When $n=3$ and $0<a_{2}<1$, then we have

$$
\frac{E^{\prime}\left(a_{2}\right)}{2 \omega_{2}}=a_{2}-A_{3}-A_{3}^{(1-q) / q}=0
$$

Hence,

$$
\begin{aligned}
E_{3}\left(a_{2}\right) & =M_{3,2}^{2}-A_{3}^{2}-2 q A_{3}^{1 / q} \\
& =M_{3,2}^{2}+(1-2 q) A_{3}^{1 / q}-\left(A_{3}^{2}+A_{3}^{1 / q}\right) \\
& =M_{3,2}^{2}+(1-2 q) A_{3}^{1 / q}-a_{2} A_{3} \\
& =\omega_{3}\left(1-a_{2}\right)+(1-2 q) A_{3}^{1 / q}>0 .
\end{aligned}
$$

So we only need to consider the case $n=2$ and $E_{2} \geq 0$ is equivalent to $g(q)=(1-$ $q) / 2-q^{1 / q} \geq 0$ and $(1-q) / 2-(1-q)^{1 / q} \geq 0$, the second inequality follows from Lemma 2.6 and one checks that $g(q)$ is a decreasing function of $q$ so that $g(q) \geq g(1 / 2)=0$ with equality holding if and only if $q=1 / 2$. This completes the proof for the left-hand side inequality of (6.14).

For the right-hand side inequality of (6.14), we may assume $1 \leq x_{1} \leq x_{2} \leq \cdots \leq x_{n}$ and define

$$
F_{n}\left(x_{2}, \ldots, x_{n}\right)=2 q\left(A_{n}^{1 / q}-G_{n}^{1 / q}\right)-\sigma_{n} .
$$

We want to show $F_{n} \geq 0$. Let $\mathbf{a}=\left(a_{2}, \ldots, a_{n}\right) \in[1, \infty)^{n-1}$ be the point in which the absolute minimum of $F_{n}$ is reached.

Again by Lemma 2.5 we may assume $1=a_{1}<a_{2}<\cdots<a_{n}$. If $a_{n}<\infty$, then $a$ is an interior point of $[0,1]^{n-1}$, and we obtain

$$
\nabla F_{n}\left(a_{2}, \ldots, a_{n}\right)=0
$$

such that $a_{2}, \ldots, a_{n}$ solve (6.17), which has at most two roots (regarding $A_{n}, G_{n}$ as constants), so we are reduced to the case $n=3$. But if $1<a_{2}<a_{3}$, then we will have (6.18), which is again impossible.

Thus we only need to consider the case $n=2$ and $F_{2} \geq 0$ follows similarly to our treatment of $D_{2} \geq 0$. One checks easily here $F_{2}\left(x_{2}\right)=0$ if and only if $x_{2}=1$ or $q=1 / 2$. 
So now we only need to consider the case $a_{n} \rightarrow \infty$. In this case it is easy to see that if $q=1 / 2$, then $n=2$ and $F_{2}=0$. If $q<1 / 2$, then $1 / q>2$, hence

$$
\lim _{a_{n} \rightarrow \infty} F_{n}=\lim _{a_{n} \rightarrow \infty} a_{n}^{1 / q} \cdot \lim _{a_{n} \rightarrow \infty} \frac{2 q A_{n}^{1 / q}}{a_{n}^{1 / q}}>0 .
$$

This now completes the proof for the right-hand side inequality of (6.1) with the conditions for equality readily checked.

\section{Results on symmetric means}

Our key tool in studying the symmetric means is Lemma 2.7. We remark here that it follows from the proof of the lemma (see, e.g., [2, pages 317-318]) that for any $t \geq 0$, $P_{n, i}\left(\mathbf{x}_{t}\right)=P_{r, i}\left(\mathbf{y}_{t}\right)$. For an application of the lemma, we note the following result (see [15, Theorems 51 and 52], but be aware of the changes in notation).

TheORem 7.1. For $n \geq 1$,

$$
P_{n, 1} \geq P_{n, 2} \geq \cdots \geq P_{n, n-1} \geq P_{n, n}
$$

and, for $n>r>0$,

$$
P_{n, r}^{2 r} \geq P_{n, r-1}^{r-1} P_{n, r+1}^{r+1} .
$$

In [15], it shows that (7.2) implies (7.1). We now use Lemma 2.7 to show that the two are equivalent.

Theorem 7.2. Inequalities (7.1), (7.2), and $P_{n, 1} \geq P_{n, n}$ are equivalent.

Proof. Plainly (7.1) implies $P_{n, 1} \geq P_{n, n}$ and via a change of variables $x_{i} \rightarrow 1 / x_{n-i+1}, P_{n, 1} \geq$ $P_{n, n}$ is equivalent to $P_{n, n-1} \geq P_{n, n}$ and then Lemma 2.7 gives (7.1). To show (7.2) implies (7.1), we let $f(t)=\ln \left(P_{n, r}\left(\mathbf{x}_{t}\right) / P_{n, r+1}\left(\mathbf{x}_{t}\right)\right)$ and note that $f^{\prime}(t) \leq 0$ implies (7.1) since $\lim _{t \rightarrow \infty} f(t)=0$. As $\mathbf{x}$ is arbitrary, it suffices to show $f^{\prime}(0) \leq 0$, which is equivalent to (7.2). Now we show that (7.2) follows from (7.1). For a given $\mathbf{x}=\left(x_{1}, \ldots, x_{n}\right)$, we define $\mathbf{x}^{-1}=\left(1 / x_{1}, \ldots, 1 / x_{n}\right)$. Note $P_{n, j}^{j}\left(\mathbf{x}^{-1}\right)=P_{n, n-j}^{n-j}(\mathbf{x}) / P_{n, n}^{n}(\mathbf{x})$. Hence, (7.1) implies $P_{n, 1}\left(\mathbf{x}^{-1}\right) \geq$ $P_{n, 2}\left(\mathbf{x}^{-1}\right)$ or $P_{n, n-1}^{2(n-1)} \geq P_{n, n-2}^{n-2} P_{n, n}^{n}$. This combined with Lemma 2.7 gives (7.2).

Our next result generalizes (1.8).

Theorem 7.3. For $t \geq 0, n \geq r \geq 2$,

$$
\begin{gathered}
\frac{\sigma_{n}}{2(n-1) x_{1}^{2}} \geq \ln P_{n, r-1}-\ln P_{n, r} \geq \frac{\sigma_{n}}{2(n-1) x_{n}^{2}}, \\
\frac{r \sigma_{n}}{2(n-1) x_{1}^{2-r /(r-1)}} \geq P_{n, 1}^{r /(r-1)}-P_{n, r}^{r /(r-1)} \geq \frac{r \sigma_{n}}{2(n-1) x_{n}^{2-r /(r-1)},} \\
\left(\frac{x_{n}}{t+x_{n}}\right)^{r-2}\left(P_{n, 1, t}^{r}-P_{n, r, t}^{r}\right) \geq P_{n, 1}^{r}-P_{n, r}^{r} \geq\left(\frac{x_{1}}{t+x_{1}}\right)^{r-2}\left(P_{n, 1, t}^{r}-P_{n, r, t}^{r}\right), \\
\frac{r(r-1) x_{n}^{r-2}}{2(n-1)} \sigma_{n} \geq P_{n, 1}^{r}-P_{n, r}^{r} \geq \frac{r(r-1) x_{1}^{r-2}}{2(n-1)} \sigma_{n} .
\end{gathered}
$$


Proof. We note first $\sigma_{n}=(n-1)\left(P_{n, 1}^{2}-P_{n, 2}^{2}\right)$. By Lemma 2.7, there exists $\mathbf{y}=\left(y_{1}, \ldots, y_{r}\right)$ with $x_{1} \leq y_{1} \leq \cdots \leq y_{r} \leq x_{n}$ such that $P_{n, i}(\mathbf{x})=P_{r, i}(\mathbf{y}), 0 \leq i \leq r$. Further note that $P_{r, r}(\mathbf{y})=G_{r}, P_{r, r-1}(\mathbf{y})=G_{r}^{r /(r-1)} / H_{r}^{1 /(r-1)}$. By a result of the author [13, (3.5)] and using the same method that shows that (1.2) and (1.3) are equivalent, one can show that

$$
\frac{P_{r, 1}^{2}(\mathbf{y})-P_{r, 2}^{2}(\mathbf{y})}{2 y_{1}^{2}} \geq \ln P_{r, r-1}(\mathbf{y})-\ln P_{r, r}(\mathbf{y}) \geq \frac{P_{r, 1}^{2}(\mathbf{y})-P_{r, 2}^{2}(\mathbf{y})}{2 y_{r}^{2}} .
$$

Inequality (7.3) then follows from this and Lemma 2.7. Similarly, (7.4) follows from Theorem 6.1 and Lemma 2.7. To show (7.5), we use an identity of Dinghas [11, (3.3)]:

$$
A_{n}^{r}-P_{n, r}^{r}=\frac{1}{\left(\begin{array}{l}
n \\
r
\end{array}\right)} \sum_{k=2}^{n} \sum_{i=2}^{k}(i-1) \frac{\left(x_{k}-A_{k-1}\right)^{2}}{k^{2}} E_{n-2, r-2}^{i-2, k-i}\left(A_{k-1} ; A_{k} ; x_{k+1}, \ldots, x_{n}\right),
$$

where $E_{n-2, r-2}^{i-2, k-i}\left(A_{k-1} ; A_{k} ; x_{k+1}, \ldots, x_{n}\right)$ denotes the $(r-2)$ th symmetric function of the $n-$ 2 numbers $A_{k-1}$ ( $i-2$ times), $A_{k}$ ( $k-i$ times) and $x_{k+1}, \ldots, x_{n}$.

Now use (7.8) for $\left(A_{n}^{r}-P_{n, r}^{r}\right) / x_{n}^{r-2}$ and $\left(A_{n, t}^{r}-P_{n, r, t}^{r}\right) /\left(x_{n}+t\right)^{r-2}$ and consider their differences, the left-hand side inequality of (7.5) follows from this and the observation

$$
\frac{x_{i}+t}{x_{n}+t} \geq \frac{x_{i}}{x_{n}}, \quad 1 \leq i \leq n, \quad \frac{A_{i}+t}{x_{n}+t} \geq \frac{A_{i}}{x_{n}}, \quad i=k-1, k .
$$

The right-hand side inequality of (7.5) can be shown similarly. Now (7.6) follows from (7.5). This can be seen by noticing $\lim _{t \rightarrow \infty}\left(x_{1}+t\right)^{2-r}\left(A_{n, t}^{r}-P_{n, r, t}^{r}\right)=\lim _{t \rightarrow \infty}\left(x_{n}+t\right)^{2-r}\left(A_{n, t}^{r}\right.$ $\left.-P_{n, r, t}^{r}\right)=r(r-1) \sigma_{n} / 2(n-1)$ and this completes the proof.

We note that (7.6) is a result of Dinghas [11, page 156], originally written as

$$
\begin{aligned}
& \frac{r(r-1) x_{n}^{r-2}}{2 n(n-1)} \sum_{k=1}^{n}\left(1-\frac{1}{k}\right)\left(x_{k}-A_{k-1}\right)^{2} \\
& \quad \geq A_{n}^{r}-P_{n, r}^{r} \geq \frac{r(r-1) x_{1}^{r-2}}{2 n(n-1)} \sum_{k=1}^{n}\left(1-\frac{1}{k}\right)\left(x_{k}-A_{k-1}\right)^{2} .
\end{aligned}
$$

By using the relation $(k-1) A_{k-1}+x_{k}=k A_{k}$, one shows easily by induction that $\sum_{k=1}^{n}(1-$ $1 / k)\left(x_{k}-A_{k-1}\right)^{2}=n \sigma_{n}$ and (7.6) then follows.

Corollary 7.4. For $n \geq r \geq 2$,

$$
r x_{n}\left(A_{n}^{r-1}-P_{n, r-1}^{r-1}\right) \geq(r-2)\left(A_{n}^{r}-P_{n, r}^{r}\right) \geq r x_{1}\left(A_{n}^{r-1}-P_{n, r-1}^{r-1}\right) .
$$

Proof. Let $f(t)=\left(x_{n}+t\right)^{2-r}\left(A_{n, t}^{r}-P_{n, r, t}^{r}\right)$. By (7.5), $f$ is an increasing function of $t$ and $f^{\prime}(0) \geq 0$ gives the left-hand inequality of (7.11) and the right-hand inequality of (7.11) follows similarly. 
Theorem 7.5. For $t \geq 0, n-1 \geq r \geq 1$,

$$
\left(\frac{x_{n}}{t+x_{n}}\right)^{2 r-2} \geq \frac{P_{n, r}^{2 r}-P_{n, r-1}^{r-1} P_{n, r+1}^{r+1}}{P_{n, r, t}^{2 r}-P_{n, r-1, t}^{r-1} P_{n, r+1, t}^{r+1}} \geq\left(\frac{x_{1}}{t+x_{1}}\right)^{2 r-2} .
$$

Proof. The proof is similar to the proof of Theorem 7.3, once we note the following identity of Muirhead [21] (see also [15, Theorem 54]):

$$
P_{n, r}^{2 r}-P_{n, r-1}^{r-1} P_{n, r+1}^{r+1}=\left(r(r+1)\left(\begin{array}{c}
n \\
r
\end{array}\right)\left(\begin{array}{c}
n \\
r+1
\end{array}\right)\right) \sum_{i=0}^{-1}\left(\begin{array}{c}
2 i \\
i
\end{array}\right) \frac{(r, i)}{i+1},
$$

where $(r, i)=\sum x_{1}^{2} \cdots x_{r-i-1}^{2} x_{r-i} \cdots x_{r+i-1}\left(x_{r+i}-x_{r+i+1}\right)^{2}$, the summation extending over all products formed from the $\mathbf{x}$ and of the type shown.

We note here that by taking the limit as $t \rightarrow \infty$ in Theorem 7.5 , we obtain

$$
\frac{x_{n}^{2 r-2}}{(n-1)} \sigma_{n} \geq P_{n, r}^{2 r}-P_{n, r-1}^{r-1} P_{n, r+1}^{r+1} \geq \frac{x_{1}^{2 r-2}}{(n-1)} \sigma_{n} .
$$

We leave the proof of the following corollary to the reader since it is similar to the proof of Corollary 7.4.

Corollary 7.6. For $n-1 \geq r \geq 2$,

$$
\begin{aligned}
x_{n}\left(P_{n, r}^{r} P_{n, r-1}^{r-1}-P_{n, r-2}^{r-2} P_{n, r+1}^{r+1}\right) & \geq 2\left(P_{n, r}^{2 r}-P_{n, r-1}^{r-1} P_{n, r+1}^{r+1}\right) \\
& \geq x_{1}\left(P_{n, r}^{r} P_{n, r-1}^{r-1}-P_{n, r-2}^{r-2} P_{n, r+1}^{r+1}\right) .
\end{aligned}
$$

We now look at the following inequalities in the unweighted case $\left(\omega_{i}=1 / n, n \geq 2\right)$ :

$$
\begin{aligned}
& (n-1)\left(M_{n, 2}^{2}-A_{n}^{2}\right) \geq A_{n}^{2}-G_{n}^{2} \geq \frac{1}{n-1}\left(M_{n, 2}^{2}-A_{n}^{2}\right), \\
& (n-1)\left(M_{n, 2}^{2}-A_{n}^{2}\right) \geq A_{n}^{2}-A_{n} H_{n} \geq \frac{1}{n-1}\left(M_{n, 2}^{2}-A_{n}^{2}\right) .
\end{aligned}
$$

Inequality (7.16) is due to Diananda [10]. Inequality (7.17) follows from Corollary 5.3.

We note that the two left-hand side inequalities of (7.16) and (7.17) give refinements of (7.2). Since $M_{n, 2}^{2}-A_{n}^{2}=(n-1)\left(P_{n, 1}^{2}-P_{n, 2}^{2}\right)$, the left-hand side inequality of (7.16) is equivalent to $(n-1)^{2} P_{n, 2}^{2} \leq n(n-2) P_{n, 1}^{2}+P_{n, n}^{2}$. By a change of variables $x_{i} \rightarrow 1 / x_{n-i+1}$, this is

$$
(n-1)^{2} P_{n, n-2}^{n-2} P_{n, n}^{n} \leq n(n-2) P_{n, n-1}^{2 n-2}+P_{n, n}^{2 n-2} .
$$

It follows then from Lemma 2.7 that for $2 \leq r \leq n$,

$$
(r-1)^{2} P_{n, r-2}^{r-2} P_{n, r}^{r} \leq r(r-2) P_{n, r-1}^{2 r-2}+P_{n, r}^{2 r-2} \leq(r-1)^{2} P_{n, r-1}^{2 r-2}
$$


Similarly, the left-hand side inequality of (7.17) gives for $2 \leq r \leq n$,

$$
(r-1)^{2} P_{n, r-2}^{r-2} P_{n, r}^{r} \leq r(r-2) P_{n, r-1}^{2 r-2}+P_{n, r-1}^{r-1} P_{n, r}^{r} / P_{n, 1} \leq(r-1)^{2} P_{n, r-1}^{2 r-2} .
$$

The two right-hand inequalities of (7.16) and (7.17) are relatively easy. For example, the right-hand side inequality of (7.16) is equivalent to $P_{n, 2} \geq P_{n, n}$. We now give a uniform treatment of the two right-hand side inequalities.

Theorem 7.7. For $t \geq 0, n \geq r \geq 2, f(t ; \alpha)=P_{n, 1, t}^{\alpha}-P_{n, r, t}^{\alpha}$ is a decreasing function of $t$ for $\alpha \leq r /(r-1)$ and $P_{n, 1, t}^{\alpha}-P_{n, r, t}^{\alpha}$ is an increasing function for $\alpha \geq r$. In particular, for $n \geq 3$, $\omega_{i}=1 / n$, one has

$$
(1-1 / n) \frac{G_{n}^{n /(n-2)} A_{n}^{(n-3) /(n-2)}}{H_{n}^{1 /(n-2)}}+\frac{M_{n, 2}^{2}}{n} \leq A_{n}^{2} .
$$

Proof. The first assertion of the theorem follows from Corollary 5.4 and Lemma 2.7. Apply this to $r=n-1$ so that $f^{\prime}(0 ;(n-1) /(n-2)) \leq 0 ;(7.21)$ follows from this by a change of variables $x_{i} \rightarrow 1 / x_{n-i+1}$.

Note that when $n \geq 3$ and by the well-known Sierpiński inequality $A_{n} H_{n}^{n-1} \leq G_{n}^{n}$,

$$
\max \left(G_{n}^{2}, A_{n} H_{n}\right) \leq \frac{G_{n}^{n /(n-2)} A_{n}^{(n-3) /(n-2)}}{H_{n}^{1 /(n-2)}} .
$$

Hence, (7.21) gives a refinement of the right-hand side inequalities of (7.16) and (7.17).

Our next result generalizes Theorem 1.4 for $k=1$.

Theorem 7.8. For $n \geq r \geq 1$, inequality

$$
(r+1)\left(\ln \left(\frac{P_{n+1,1}}{P_{n+1,1, t}}\right)-\ln \left(\frac{P_{n+1, r+1}}{P_{n+1, r+1, t}}\right)\right) \geq r\left(\ln \left(\frac{P_{n, 1}}{P_{n, 1, t}}\right)-\ln \left(\frac{P_{n, r}}{P_{n, r, t}}\right)\right)
$$

holds with equality holding if and only if $x_{1}=\cdots=x_{n}$ when $r \neq n$ and $x_{n+1}=A_{n}$ when $r=n$.

Proof. We use the idea in [8] and we may assume $n>r$. In this case, (7.23) is equivalent to

$$
\left(\frac{P_{n+1, r+1}^{r+1} / P_{n, r}^{r}}{P_{n+1, r+1, t}^{r+1} / P_{n, r, t}^{r}}\right)\left(\frac{P_{n, 1}}{P_{n, 1, t}}\right)^{r} \leq\left(\frac{P_{n+1,1}}{P_{n+1,1, t}}\right)^{r+1}
$$

Using the relation

$$
P_{n+1, r+1}^{r+1}=\frac{n-r}{n+1} P_{n, r+1}^{r+1}+\frac{r+1}{n+1} x_{n+1} P_{n, r}^{r}
$$

we can express the first factor on the left-hand side of (7.24) as

$$
\frac{(n-r) P_{n, r+1}^{r+1} / P_{n, r}^{r}+(r+1) x_{n+1}}{(n-r) P_{n, r+1, t}^{r+1} / P_{n, r, t}^{r}+(r+1)\left(x_{n+1}+t\right)} .
$$


3572 A new approach to Ky Fan-type inequalities

Similarly, by Theorem 7.7 with $\alpha=0$,

$$
\begin{aligned}
\left(\frac{P_{n+1,1}}{P_{n+1,1, t}}\right)^{r+1} & =\left(\frac{P_{n+1,1}\left(x_{n+1}, P_{n, 1}, \ldots, P_{n, 1}\right)}{P_{n+1,1}\left(x_{n+1}+t, P_{n, 1}+t, \ldots, P_{n, 1}+t\right)}\right)^{r+1} \\
& \geq\left(\frac{P_{n+1, r+1}\left(x_{n+1}, P_{n, 1}, \ldots, P_{n, 1}\right)}{P_{n+1, r+1, t}\left(x_{n+1}+t, P_{n, 1}+t, \ldots, P_{n, 1}+t\right)}\right)^{r+1} \\
& =\frac{(n-r) P_{n, 1}^{r+1}+(r+1) x_{n+1} P_{n, 1}^{r}}{(n-r) P_{n, 1, t}^{r+1}+(r+1)\left(x_{n+1}+t\right) P_{n, 1, t}^{r}} .
\end{aligned}
$$

Thus our conclusion will follow provided that

$$
\frac{(n-r) P_{n, r+1}^{r+1} / P_{n, r}^{r}+(r+1) x_{n+1}}{(n-r) P_{n, r+1, t}^{r+1} / P_{n, r, t}^{r}+(r+1)\left(x_{n+1}+t\right)} \leq \frac{(n-r) P_{n, 1}+(r+1) x_{n+1}}{(n-r) P_{n, 1, t}+(r+1)\left(x_{n+1}+t\right)} .
$$

If $x_{1}=\cdots=x_{n}$, then equality holds above, otherwise little calculation shows that the above is equivalent to

$$
\frac{P_{n, 1}-P_{n, r+1}^{r+1} / P_{n, r}^{r}}{P_{n, 1, t}-P_{n, r+1, t}^{r+1} / P_{n, r, t}^{r}}-1 \geq \frac{-t(n+1)}{(n-r) P_{n, 1, t}+(r+1)\left(x_{n+1}+t\right)} .
$$

In order for the above inequality to hold, it suffices to show that the left-hand side above $\geq 0$ and this last inequality follows by using the method in the proof of Theorem 7.3 combined with the case $s=-1$ in Corollary 3.3 and Lemma 2.7.

Corollary 7.9. For $n \geq r \geq 1$,

$$
(r+1)\left(\frac{P_{n+1, r}^{r}}{P_{n+1, r+1}^{r+1}}-\frac{1}{A_{n+1}}\right) \geq r\left(\frac{P_{n, r-1}^{r-1}}{P_{n, r}^{r}}-\frac{1}{A_{n}}\right) .
$$

Proof. It follows from Theorem 7.8 that the function $f(t)=(r+1)\left(\ln A_{n+1, t}-\ln P_{n+1, r+1, t}\right)$ $-r\left(\ln A_{n, t}-\ln P_{n, r, t}\right)$ is a decreasing function of $t$ and the conclusion follows from $f^{\prime}(0) \leq$ 0 .

Our last result is analogous to Theorem 1.4 and the classical Rado inequality.

Theorem 7.10. For $n \geq r \geq 2$,

$$
n\left(P_{n, 1}-P_{n, r}\right) \geq(n-1)\left(P_{n-1,1}-P_{n-1, r-1}\right)
$$

with equality holding in and only if $r=n$ and $x_{i}=P_{n-1, n-1}\left(x_{1}, \ldots, x_{i-1}, x_{i+1}, \ldots, x_{n}\right)$ for some $1 \leq i \leq n$.

Proof. We assume here that $x_{i}>0,1 \leq i \leq n$, and they satisfy no other relations. Now fix $x_{1}, \ldots, x_{n-1}$ and by using $(7.25)$, consider

$$
f\left(x_{n}\right):=n\left(P_{n, 1}-P_{n, r}\right)=x_{n}+(n-1) P_{n-1,1}-n\left(\frac{n-r}{n} P_{n-1, r}^{r}+\frac{r}{n} x_{n} P_{n-1, r-1}^{r-1}\right)^{1 / r} .
$$


On setting $f^{\prime}\left(x_{n}\right)=0$, we obtain

$$
\left(\frac{n-r}{n} P_{n-1, r}^{r}+\frac{r}{n} x_{n} P_{n-1, r-1}^{r-1}\right)^{(1-r) / r}=P_{n-1, r-1}^{1-r} .
$$

From this we solve that

$$
x_{n}=\frac{n}{r} P_{n-1, r-1}-\frac{n-r}{r} \frac{P_{n-1, r}^{r}}{P_{n-1, r-1}^{r-1}}>0 .
$$

Since one checks easily that $f^{\prime \prime}\left(x_{n}\right)>0$ at the above point, it follows that for any $x_{n}>0$,

$$
\begin{aligned}
f\left(x_{n}\right) & \geq f\left(\frac{n}{r} P_{n-1, r-1}-\frac{n-r}{r} \frac{P_{n-1, r}^{r}}{P_{n-1, r-1}^{r-1}}\right) \\
& =\frac{n}{r} P_{n-1, r-1}-\frac{n-r}{r} \frac{P_{n-1, r}^{r}}{P_{n-1, r-1}^{r-1}}+(n-1) P_{n-1,1}-n P_{n-1, r-1} \\
& =(n-1)\left(P_{n-1,1}-P_{n-1, r-1}\right)+\frac{n-r}{r}\left(P_{n-1, r-1}-\frac{P_{n-1, r}^{r}}{P_{n-1, r-1}^{r-1}}\right) \\
& \geq(n-1)\left(P_{n-1,1}-P_{n-1, r-1}\right),
\end{aligned}
$$

where the last inequality above follows from (7.1) and this proves the assertion of the lemma. One also checks the conditions for equality easily.

\section{References}

[1] J. Aczél and Zs. Páles, The behaviour of means under equal increments of their variables, Amer. Math. Monthly 95 (1988), no. 9, 856-860.

[2] H. Alzer, The inequality of Ky Fan and related results, Acta Appl. Math. 38 (1995), no. 3, 305354.

[3] A new refinement of the arithmetic mean - geometric mean inequality, Rocky Mountain J. Math. 27 (1997), no. 3, 663-667.

[4] Some inequalities for arithmetic and geometric means, Proc. Roy. Soc. Edinburgh Sect. A 129 (1999), no. 2, 221-228.

[5] H. Alzer, S. Ruscheweyh, and L. Salinas, On Ky Fan-type inequalities, Aequationes Math. 62 (2001), no. 3, 310-320.

[6] E. F. Beckenbach, A class of mean value functions, Amer. Math. Monthly 57 (1950), no. 1, 1-6.

[7] J. L. Brenner and B. C. Carlson, Homogeneous mean values: weights and asymptotics, J. Math. Anal. Appl. 123 (1987), no. 1, 265-280.

[8] P. S. Bullen and M. Marcus, Symmetric means and matrix inequalities, Proc. Amer. Math. Soc. 12 (1961), no. 2, 285-290.

[9] D. I. Cartwright and M. J. Field, A refinement of the arithmetic mean-geometric mean inequality, Proc. Amer. Math. Soc. 71 (1978), no. 1, 36-38.

[10] P. H. Diananda, On some inequalities of H. Kober, Proc. Cambridge Philos. Soc. 59 (1963), 341-346.

[11] A. Dinghas, Some identities between arithmetic means and the other elementary symmetric functions of n numbers, Math. Ann. 120 (1948), no. 1, 154-157.

[12] Superadditive Funktionale, Identitäten und Ungleichungen der elementaren Analysis, Math. Ann. 178 (1968), no. 4, 315-334. 


\section{A new approach to Ky Fan-type inequalities}

[13] P. Gao, Certain bounds for the difference of means, JIPAM. J. Inequal. Pure Appl. Math. 4 (2003), no. 4 , article $76,10 \mathrm{pp}$.

[14] Ky Fan inequality and bounds for differences of means, Int. J. Math. Math. Sci. 2003 (2003), no. 16, 995-1002.

[15] G. H. Hardy, J. E. Littlewood, and G. Pólya, Inequalities, 2nd ed., Cambridge University Press, Cambridge, 1952.

[16] L. Hoehn and I. Niven, Averages on the move, Math. Mag. 58 (1985), no. 3, 151-156.

[17] A. McD. Mercer, Some new inequalities involving elementary mean values, J. Math. Anal. Appl. 229 (1999), no. 2, 677-681.

[18] Bounds for $A-G, A-H, G-H$, and a family of inequalities of Ky Fan's type, using a general method, J. Math. Anal. Appl. 243 (2000), no. 1, 163-173.

[19] - Improved upper and lower bounds for the difference $A_{n}-G_{n}$, Rocky Mountain J. Math. 31 (2001), no. 2, 553-560.

[20] P. R. Mercer, Refined arithmetic, geometric and harmonic mean inequalities, Rocky Mountain J. Math. 33 (2003), no. 4, 1459-1464.

[21] R. F. Muirhead, Inequalities relating to some algebraic means, Proc. Edinburgh Math. Soc. 19 (1901), 36-45.

[22] J. E. Pečarić and H. Alzer, On Ky Fan's inequality, Math. Pannon. 6 (1995), no. 1, 85-93, Festschrift for Hans Vogler on the occasion of his 60th birthday.

[23] W. Sierpiński, On an inequality for arithmetic, geometric and harmonic means, Warsch. Sitzungsber. 2 (1909), 354-358 (Polish).

[24] W. L. Wang and P. F. Wang, A class of inequalities for the symmetric functions, Acta Math. Sinica 27 (1984), no. 4, 485-497 (Chinese).

[25] C. Wu, W. L. Wang, and L. Fu, Inequalities for symmetric functions and their applications, J. Chengdu Univ. Sci. Tech. (1982), no. 1, 103-108 (Chinese).

Peng Gao: Department of Mathematics, University of Michigan, Ann Arbor, MI 48109, USA

E-mail address: penggao@umich.edu

Current address: American Institute of Mathematics, 360 Portage Avenue, Palo Alto, CA 943062244, USA

E-mail address: penggao@aimath.org 


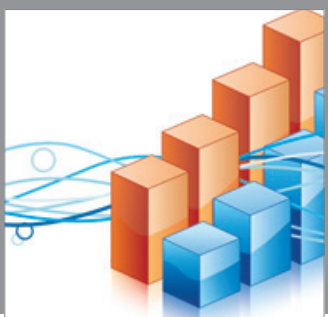

Advances in

Operations Research

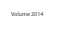

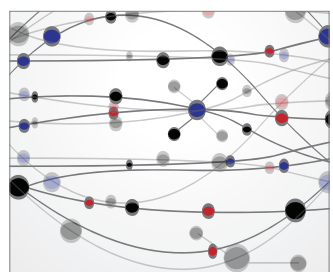

\section{The Scientific} World Journal
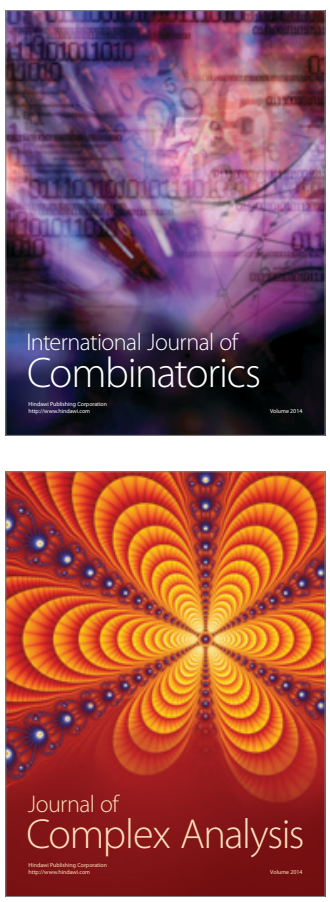

International Journal of

Mathematics and

Mathematical

Sciences
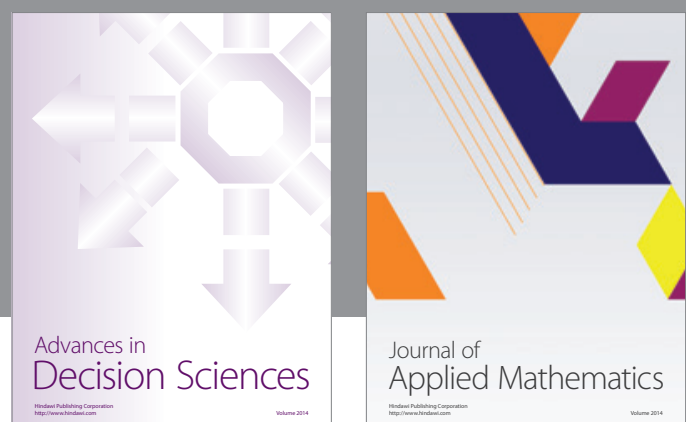

Journal of

Applied Mathematics
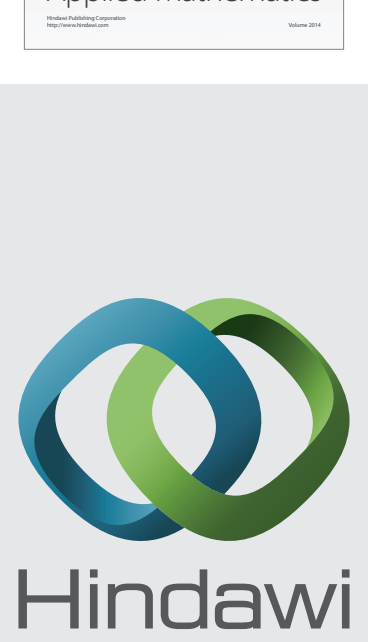

Submit your manuscripts at http://www.hindawi.com
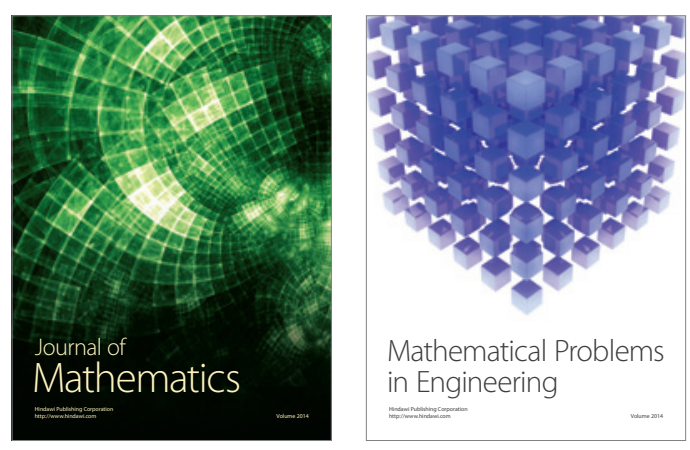

Mathematical Problems in Engineering
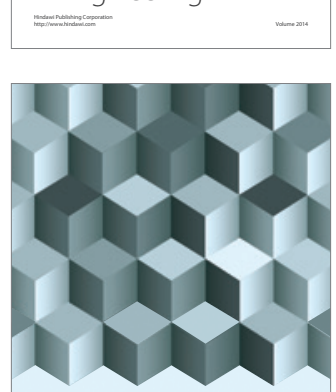

Journal of

Function Spaces
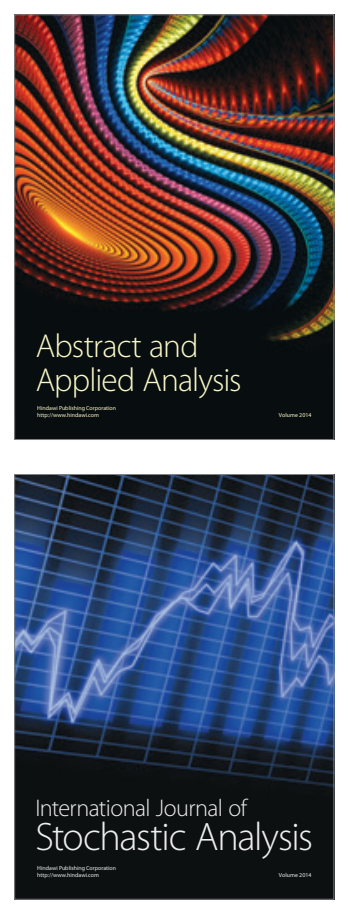

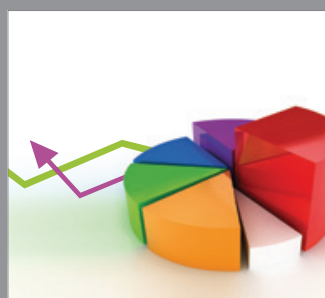

ournal of

Probability and Statistics

Promensencen
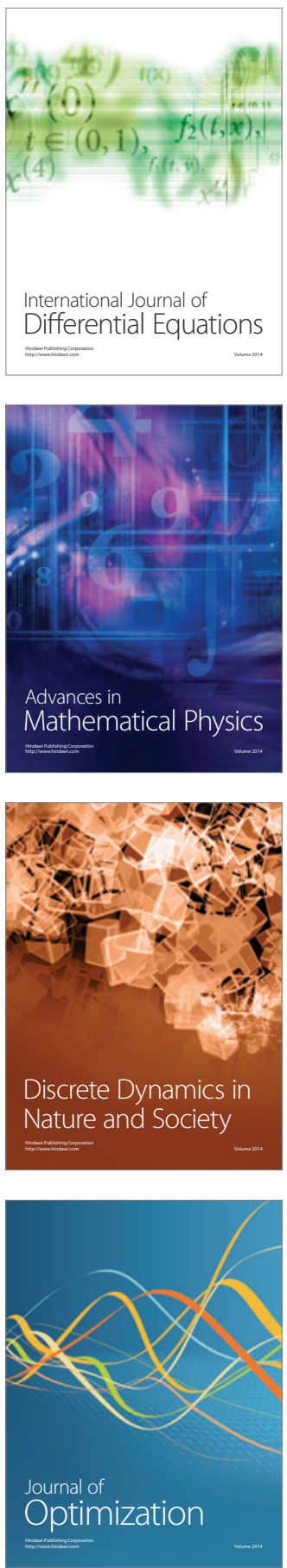